ARTICLE

https://doi.org/10.1038/s41467-019-09299-3

\title{
The conserved NxNNWHW motif in Aha-type co-chaperones modulates the kinetics of Hsp90 ATPase stimulation
}

Rebecca Mercier ${ }^{1}$, Annemarie Wolmarans ${ }^{1}$, Jonathan Schubert ${ }^{2}$, Hannes Neuweiler (1) ${ }^{2}$, Jill L. Johnson (D) ${ }^{3} \&$ Paul LaPointe ${ }^{1}$

Hsp90 is a dimeric molecular chaperone that is essential for the folding and activation of hundreds of client proteins. Co-chaperone proteins regulate the ATP-driven Hsp90 client activation cycle. Aha-type co-chaperones are the most potent stimulators of the Hsp90 ATPase activity but the relationship between ATPase regulation and in vivo activity is poorly understood. We report here that the most strongly conserved region of Aha-type co-chaperones, the $N$ terminal NxNNWHW motif, modulates the apparent affinity of Hsp90 for nucleotide substrates. The ability of yeast Aha-type co-chaperones to act in vivo is ablated when the $N$ terminal NxNNWHW motif is removed. This work suggests that nucleotide exchange during the Hsp90 functional cycle may be more important than rate of catalysis.

\footnotetext{
${ }^{1}$ Department of Cell Biology, Faculty of Medicine and Dentistry, University of Alberta, Edmonton, AB T6G 2H7, Canada. ${ }^{2}$ Department of Biotechnology and Biophysics, University of Würzburg, Würzburg 97074, Germany. ${ }^{3}$ Department of Biological Sciences and the Center for Reproductive Biology, University of Idaho, Moscow, ID 83844, USA. Correspondence and requests for materials should be addressed to P.L. (email: paul.lapointe@ualberta.ca)
} 
T he $90 \mathrm{kDa}$ heat shock protein (Hsp90) is a dimeric molecular chaperone that promotes the folding and maturation of a large but specific group of substrates called client proteins $^{1,2}$. Client activation during the Hsp90 functional cycle is regulated by a cohort of proteins called co-chaperones ${ }^{3-13}$. Cochaperones regulate conformational transitions in Hsp90, ATP binding and hydrolysis, as well as client interaction ${ }^{14,15}$. How the Hsp90 functional cycle is regulated in the context of client maturation is poorly understood but it is clear that ATP hydrolysis is critical for efficient client maturation by Hsp90 16,17 .

The importance of the Hsp90 ATPase activity has drawn a great deal of attention to the co-chaperones that regulate it. The activator of Hsp90 ATPase, Aha1, is the most potent stimulator of the Hsp90 ATPase activity identified to date ${ }^{18,19}$. Modulating Aha1 levels, and presumably the Hsp90 ATPase activity, has been shown to alter the folding of the cystic transmembrane conductance regulator (CFTR) and its export from the $\mathrm{ER}^{20}$, kinase activation $^{21-23}$, and the activity of other clients ${ }^{21,22,24}$. The cellular activity of $\mathrm{Hsp} 90$ appears to be influenced by the relative expression levels of Ahal and other co-chaperones which are normally far less abundant than the chaperone itself ${ }^{25,26}$.

Hsp90 is highly conserved with yeast and human Hsp90 possessing $\sim 60 \%$ identity. Co-chaperone proteins are not nearly as well conserved at the level of primary sequence but many are functionally interchangeable between yeast and humans ${ }^{27-29}$. The Aha-type co-chaperones are among the more weakly conserved proteins with the yeast Ahalp and human Ahsal sharing only
$23 \%$ identity but they stimulate the ATPase activity of Hsp90 in a similar manner ${ }^{30}$. Presumably their functional conservation is linked to the sequences that they share. Certainly this is true for the highly conserved RKxK motif which is not required for interaction with Hsp90 but is necessary for robust ATPase stimulation ${ }^{19}$. Curiously, the most strongly conserved region in Aha-type co-chaperones is in the $\mathrm{N}$ terminal domain and is defined by the NxNNWHW motif (Fig. 1). This sequence is located in the first 11 amino acids of Ahalp: a region that is not resolved in the co-crystal structure of the Ahalp $\mathrm{N}$ domain in complex with the Hsp90 middle domain ${ }^{19}$. Canonical Ahalp is comprised of two domains joined by a flexible linker and yeast possess a smaller, related co-chaperone called Hchlp that lacks the $\mathrm{C}$ terminal domain (Fig. 2a). The amino acid sequence of Hchlp is $~ 35 \%$ identical to the Ahalp N domain and it shares both the RKxK and NxNNWHW motifs 19,30 .

ATP hydrolysis by Hsp90 occurs in the context of a cycle involving extensive conformational rearrangements ${ }^{31,32}$. Hsp90 is comprised of an $\mathrm{N}$ terminal nucleotide-binding domain, joined to a middle domain by a charged linker, which is in turn connected to a $\mathrm{C}$ terminal domain that is primarily responsible for dimerization ${ }^{33}$. These different domains are rearranged both in the context of the individual protomers, as well as with respect to the dimer during ATP hydrolysis ${ }^{34}$. Ahalp interacts with the Hsp90 dimer in an anti-parallel fashion where the Ahalp $\mathrm{N}$ domain interacts with the middle domain of one Hsp90 subunit and the Aha1p C domain interacts with the Hsp90

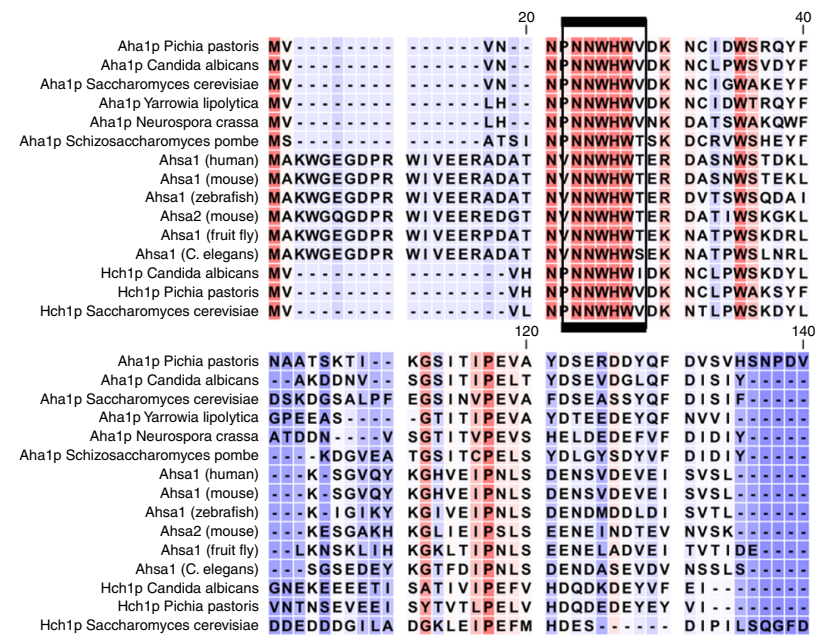

FM
KEALVGLE. KDKLLINLK.
KQKLVGVEAG
QEKLPGLEAT EDNLTKLEA

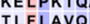

KTLFLAVR.

NGLLLGIR.

HQLFQDFK.

RELLTGFS.

QEVLPNTT -
NGKLTSLST

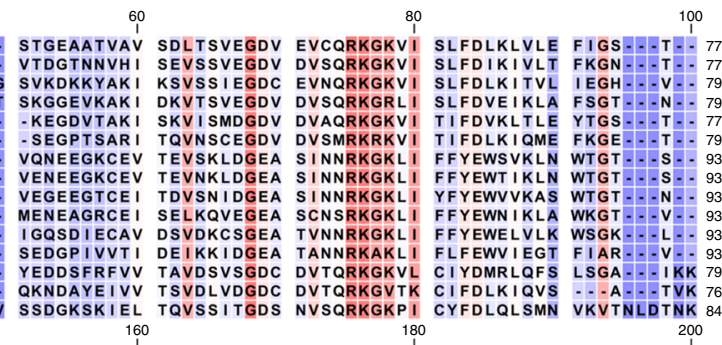

DNATEEQ- - I RALAKSKLIP QLREKLFQFG VDLIKVHGSD IQL-PAEQVK SQYTKSNQ-1 170 -NETAENSGI TDLIKKQLIP QLRTALMKF PDLIEINSKD IQL.SQDKVT STYTKANQSS 166
-KETSELSEA KPLIRSELLP KLRQIFQQFG KDLLATHGND IQV.PESQVK SNYTRGNQKS 172

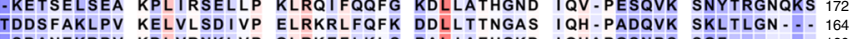
SDANEKRPV KDLVRNKLVP QLRKEFLKLS PALIAEHGKD IQHAPGSNPS SGF........

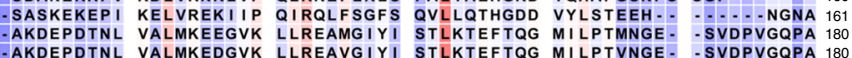

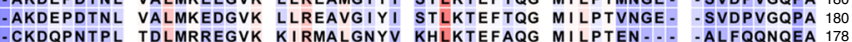

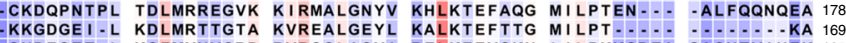

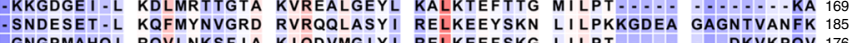
GNGPMAHQI RQVLNKSFIA KIQDVMGIYI RELKEEFSKO -E.SAS K KSQIRKLLTP ILKEKLMK AFDG...... - LVRSEFVP K K

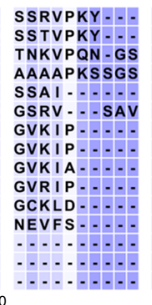
260
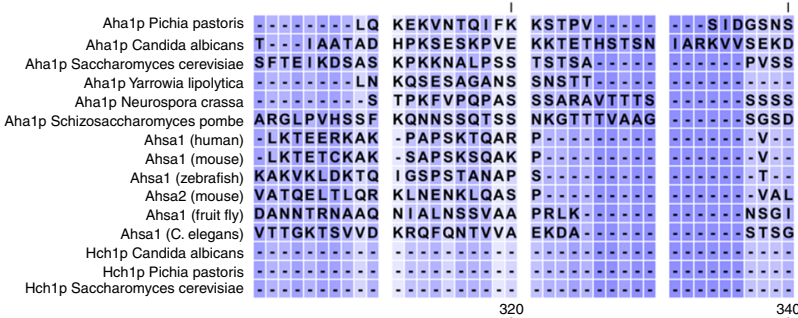
KRD
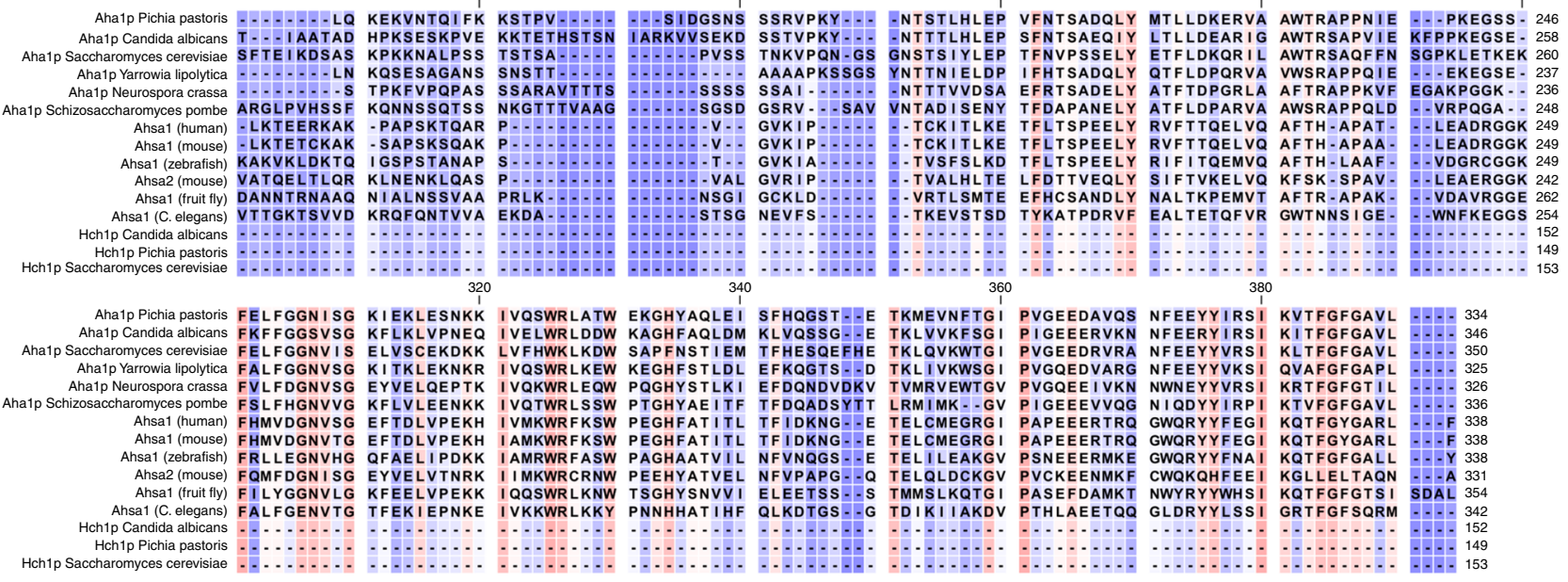

Fig. 1 Alignment of Aha-type co-chaperones. Alignment of Aha1p and Hch1p homologs shows that the NxNNWHW motif is strongly conserved across species 

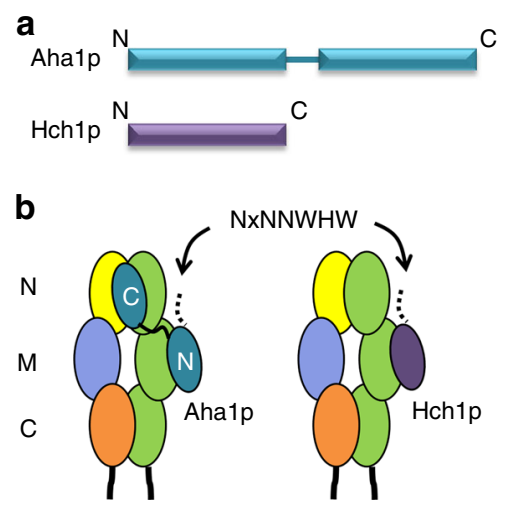

C

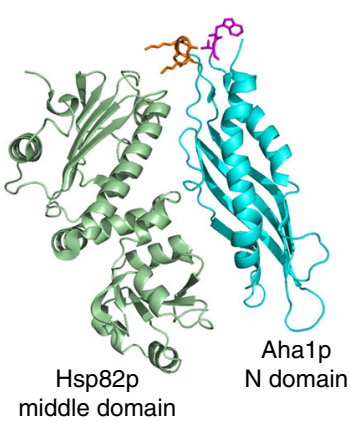

d

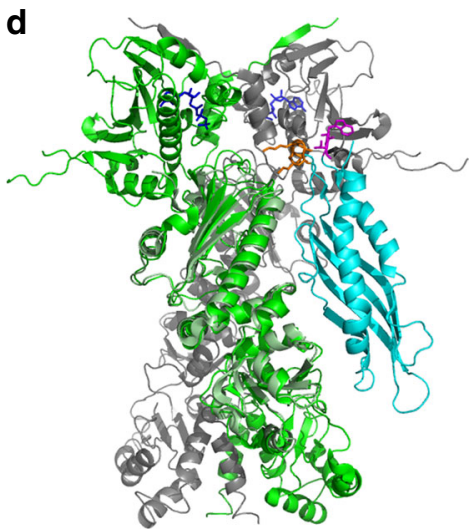

Fig. 2 Schematic of co-chaperones Aha1p and Hch1p and their interaction with Hsp90. a Hchlp corresponds to the $\mathrm{N}$ terminal domain of Aha1p which is connected to the $\mathrm{C}$ domain by a flexible linker. $\mathbf{b}$ Hch1p and the Aha1p $\mathrm{N}$ domain interact with the middle domain of Hsp90. The Aha1p $\mathrm{C}$ domain interacts with the $\mathrm{N}$ terminal domains of Hsp90. c A model of the complex between the Hsp90 middle domain (light green) and the Aha1p $\mathrm{N}$ domain (cyan) (1USV ${ }^{19}$ ). The RKxK motif is colored in orange and residues Trp11 and Val12 (magenta) indicate where the $\mathrm{N}$ terminal NxNNWHW motif would be present (it is unstructured in 1USV). $\mathbf{d}$ The structure shown in $\mathbf{c}$ is aligned to the full length, closed Hsp90 dimer structure (2CG933) with the two Sbalp subunits masked. ATP is depicted in blue wireframe. The NxNNWHW motif is predicted to be oriented towards the Hsp90 $\mathrm{N}$ domains

$\mathrm{N}$ terminal domains after they have come together in the closed conformation $^{18,19,35,36}$ (Fig. 2b). Hch1p interacts with the middle domain of $\mathrm{Hsp} 90$ in a manner similar to the Ahalp $\mathrm{N}$ domain $7,30,37,38$

Ahalp and Hch1p are important regulators of Hsp90 activity but the manner in which these co-chaperones function in vivo and the biological significance of their ATPase stimulation activities are poorly understood $7,9,13,18,19,30,35,36,39$. The NxNNWHW motif is predicted to extend towards the $\mathrm{N}$ terminus of the Hsp90 dimer based on a co-crystal structure of the Aha1p N domain and the Hsp90 middle domain ${ }^{19,33}$ (Fig. 2c, d). This proximity raises the possibility that the NxNNWHW motif could regulate conformational dynamics associated with the Hsp90 N domain and ATPase activity. In this study, we investigated the importance of the NxNNWHW motif in the biological and biochemical activities of Hch1p and Ahalp.

We report here that the NxNNWHW motif is required for the function of both Hchlp and Ahalp in vivo. Furthermore, the NxNNWHW motif is also required for optimal Hsp90 ATPase stimulation by both co-chaperones, but not for co-operative displacement of Stilp by Ahalp and Cpr6p in vitro. Strikingly, the impairment in steady-state-stimulated ATPase activity we observe is not accompanied by an impairment in the rate of conformational transitions en route to the catalytically active conformation leading us to speculate that the NxNNWHW motif could be regulating $\mathrm{Hsp} 90$ in part by mediating nucleotide exchange.

\section{Results}

The NxNNWHW motif is required for optimal ATPase stimulation. Previous work has shown that the $\mathrm{N}$ terminal 11 amino acids harboring the NxNNWHW motif are not important for Ahalp-mediated Hsp90 ATPase stimulation ${ }^{19}$. However, these experiments were carried out with $\mathrm{N}$ terminally $6 \mathrm{xHis}-$ tagged Ahalp constructs. We wondered if a potential role for the NxNNWHW motif was being masked by the $\mathrm{N}$ terminal $6 \mathrm{xHis}-$ tag. To explore this possibility we constructed two sets of Ahalp deletion mutants lacking the $\mathrm{N}$ terminal 11 amino acids encompassing the NxNNWHW motif $\left(\mathrm{Ahalp}^{\Delta 11}\right)$. One set had $\mathrm{N}$ terminal 6xHis-tags and the second had $\mathrm{C}$ terminal 6xHis-tags (Fig. 3a). We tested these co-chaperones in ATPase assays with Hsp82p and an enzymatic ATP regenerating system $7,17,30,40,41$. This assay is used to measure $V_{\max }$ under steady-state conditions with a fixed (and constant) concentration of ATP. Consistent with previous work, we found that $\mathrm{N}$ terminally 6xHis-tagged Ahalp and Ahalp $\mathrm{p}^{\Delta 11}$ stimulated Hsp82p to a similar degree (Fig. 3b). However, constructs harboring $\mathrm{C}$ terminal 6xHis-tags gave different results. Here, Ahalp ${ }^{\Delta 11}$ stimulated the ATPase activity of Hsp82p to a lesser degree than wildtype Aha1p (Fig. 3b). Surprisingly, C terminally 6xHis-tagged Ahalp stimulated ATPase activity to a far greater degree than Ahalp harboring an $\mathrm{N}$ terminal 6xHis-tag. This suggests that wildtype Ahalp function is impaired by the $\mathrm{N}$ terminal 6xHis-tag. Consequently, we used only $\mathrm{C}$ terminally tagged co-chaperone constructs in all our subsequent experiments. It is interesting to note that the apparent affinity of Ahalp ${ }^{\Delta 11}$ for Hsp82p was higher than that of wildtype Ahalp $(0.44 \pm 0.03 \mu \mathrm{M}$ compared to $1.24 \pm$ $0.06 \mu \mathrm{M}$ ) suggesting that the loss of the NxNNWHW motif stabilizes the interaction with the chaperone.

We constructed $\mathrm{C}$ terminally 6xHis-tagged wildtype Hch1p and Hch $1 \mathrm{p}^{\Delta 11}$ for testing in our ATPase-stimulation assay as well. As we observed with Ahalp, the NxNNWHW motif is important for ATPase stimulation by Hch1p (Fig. 3c). Altogether, the maximal stimulated rate of ATPase activity was $\sim 2.8$ fold lower with Ahalp $\mathrm{p}^{\Delta 11}$ compared to Ahalp, and $\sim 1.7$ fold lower with Hch1p ${ }^{\Delta 11}$ than with Hch1p. These data show that the NxNNWHW motif is required for maximal ATPase stimulation by both Hchlp and Ahalp. We also tested Ahalp ${ }^{\mathrm{N}}$ and Ahalp $\mathrm{p}^{\mathrm{N}-\Delta 11}$ in ATPase stimulation assays. Unlike the results we obtained with Hch1p, deletion of the 11 amino acids harboring the NxNNWHW motif did not impair ATPase stimulation by the Ahalp $N$ domain on its own (Fig. 3d). Importantly, we verified that deletion of the NxNNWHW motif did not alter the folding of either Ahalp or Hchlp using a ThermoFluor thermal shift assay 42,43 . The melting curves for both Aha $1 \mathrm{p}^{\Delta 11}$ and Hch $1 \mathrm{p}^{\Delta 11}$ were identical to those we obtained for their wildtype counterparts. The melting temperature $\left(T_{\mathrm{m}}\right)$ of Ahalp and Ahalp $\mathrm{p}^{\Delta 11}$ were $63.6 \pm 0.9$ and $63.3 \pm 0.9^{\circ} \mathrm{C}$, respectively. The $T_{\mathrm{m}}$ of Hch $1 \mathrm{p}$ and $\mathrm{Hch} 1 \mathrm{p}^{\Delta 11}$ were $51.5 \pm 2.0$ and $52.6 \pm 1.8^{\circ} \mathrm{C}$, respectively $(n=9)$.

Aha1p, but not Hch1p, can efficiently displace Stilp from Hsp90 in concert with Cpr6p ${ }^{9,37}$. We wondered if the NxNNWHW motif might be required for co-chaperone switching. To test this, we reconstituted an ATPase cycling reaction containing Hsp90, Stilp, Ahalp, and Cpr6 $\mathrm{p}^{37}$. Stilp is a strong inhibitor of the Hsp90 ATPase activity and of Ahalp binding to 
a

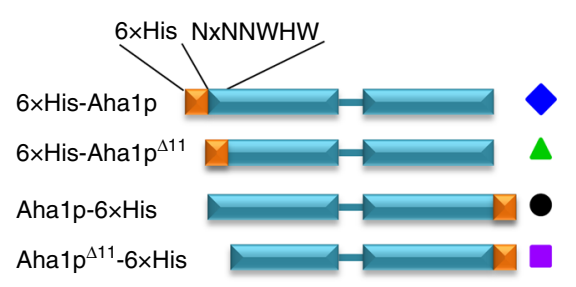

C

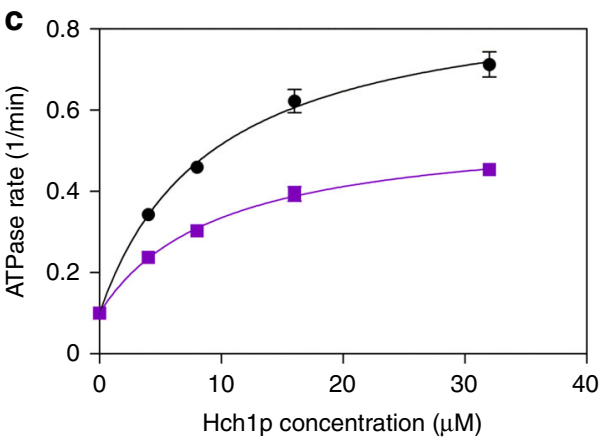

e

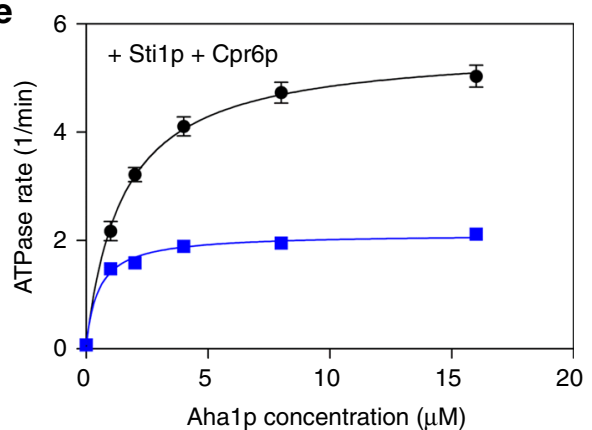

b
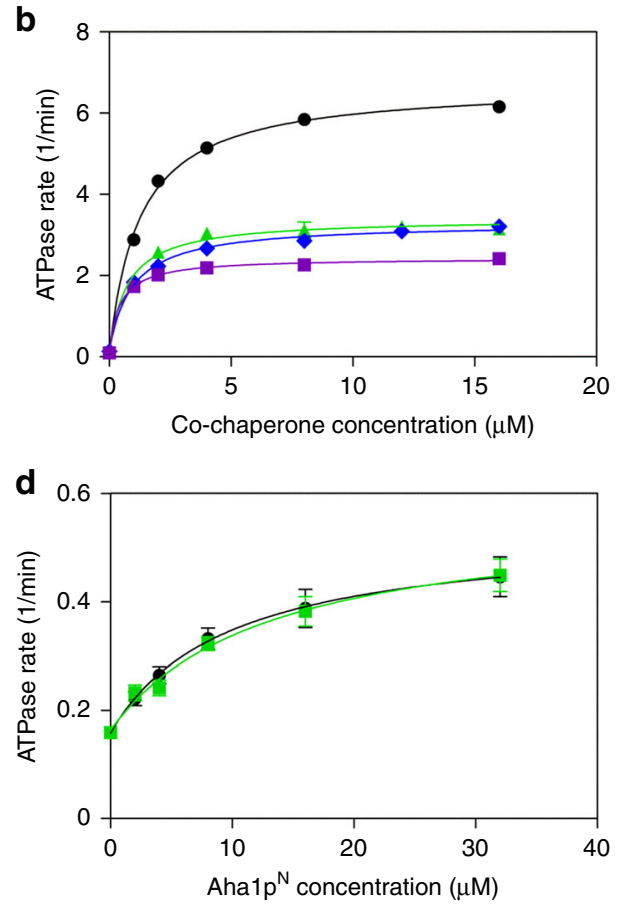

Fig. 3 Hsp90 ATPase stimulation by Aha1p and Hch1p. a Schematic of Aha1p constructs harboring $N$ and $C$ terminal $6 x$ His-tags. b Stimulation of the Hsp82p ATPase activity by increasing concentrations of Aha1p ( $\mathrm{N}$ terminal $6 \mathrm{xHis-tag-blue} \mathrm{diamonds;} \mathrm{C}$ terminal $6 \mathrm{xHis-tag-black}$ circles) and Aha1p $\Delta 11$ ( $\mathrm{N}$ terminal $6 \mathrm{xHis-tag-green} \mathrm{triangles;} \mathrm{C}$ terminal $6 \mathrm{xHis-tag-purple} \mathrm{squares).} \mathrm{Reactions} \mathrm{contained} 1 \mu \mathrm{M} \mathrm{Hsp82p}$ and indicated concentration of cochaperone $(n=3)$. c Stimulation of the Hsp82p ATPase activity by increasing concentrations of Hch1p (black circles) and Hch1p ${ }^{\Delta 11}$ (purple squares). Reactions contained $4 \mu \mathrm{M} \mathrm{Hsp82p}$ and indicated concentration of co-chaperone $(n=3)$. $\mathbf{d}$ Stimulation of the Hsp82p ATPase activity by increasing

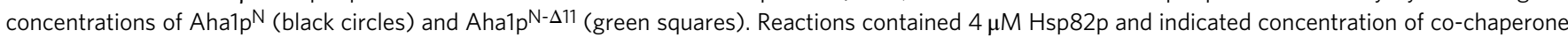
$(n=3)$. e Stimulation of the Sti1p-inhibited Hsp82p ATPase activity by increasing concentrations of Aha1p (black circles) and Aha1p ${ }^{\Delta 11}$ (blue squares) in the presence of Cpr6p. Reactions contained $1 \mu \mathrm{M} \mathrm{Hsp82p,} 4 \mu \mathrm{M}$ Sti1p, and $4 \mu \mathrm{M}$ Cpr6p $(n=3)$. Error bars show standard error of the mean

$\mathrm{Hsp} 90^{8}$. Consistent with previous reports $^{37}$, robust $\mathrm{Hsp} 90$ ATPase stimulation by Ahalp was observed in the presence of Stilp and Cpr6p (Fig. 3e). Interestingly, Ahalp $\mathrm{p}^{\Delta 11}$ was also able to stimulate Hsp90 ATPase activity in the presence of Stilp and Cpr6p suggesting that the NxNNWHW motif is not required for co-chaperone switching in vitro.

The NxNNWHW motif is required for Hchlp action in cells. The importance of the $\mathrm{N}$ terminal motif for in vivo function of Aha-type co-chaperones has never been investigated. To address this for Hch1p we employed two different yeast assays that we and other have used extensively in other studies. First, we overexpressed either Hchlp or Hchlp ${ }^{\Delta 11}$ in yeast harboring Hsp82 $\mathrm{p}^{\mathrm{E} 381 \mathrm{~K}}$ as the sole source of Hsp90 in the cell. This mutant confers slow and temperature-sensitive growth to yeast that is rescued when Hchlp, but not Ahalp, is overexpressed $7,30,44$. Hchlp overexpression rescued growth of yeast expressing
Hsp82p ${ }^{\mathrm{E} 381 \mathrm{~K}}$ but overexpression of Hch1 $\mathrm{p}^{\Delta 11}$ did not (Fig. 4a). Both Hchlp and Hch $1 p^{\Delta 11}$ were expressed in these strains and were also soluble (Fig. 4b). Second, we measured the cellular sensitivity to the Hsp90 inhibitor, NVP-AUY922, of yeast overexpressing Hch1p or Hch1 $p^{\Delta 11}$. Overexpression of Hch1p, but not Ahalp hypersensitizes yeast to Hsp90 inhibitors like NVPAUY $922^{7,30}$. This experiment revealed that deletion of the NxNNWHW motif in Hchlp eliminated the ability to induce hypersensitivity to NVP-AUY922 (Fig. 4c). Again, both Hch1p and $H c h 1 p^{\Delta 11}$ were expressed and soluble in lysates from these strains (Fig. 4d).

The NxNNWHW motif is required for Ahalp action in cells. We next wondered if the NxNNWHW motif would be required for the in vivo function of Aha1p as well. We identified a mutant of Hsc82p in a screen for HSC 82 mutants that were specifically affected by co-chaperone deletions. Yeast expressing Hsc82 $\mathrm{p}^{\mathrm{S} 25 \mathrm{P}}$ 


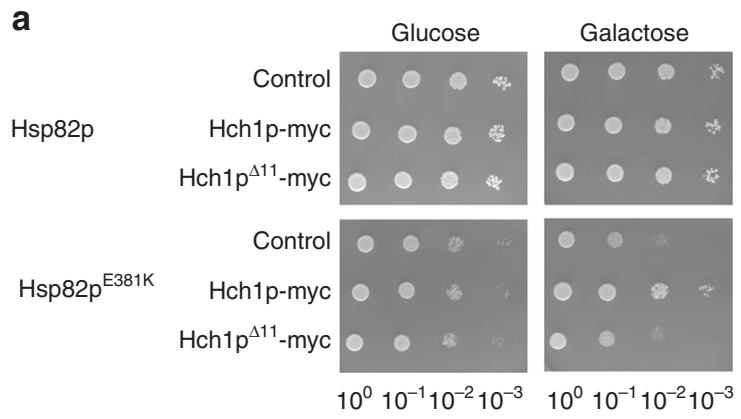

b

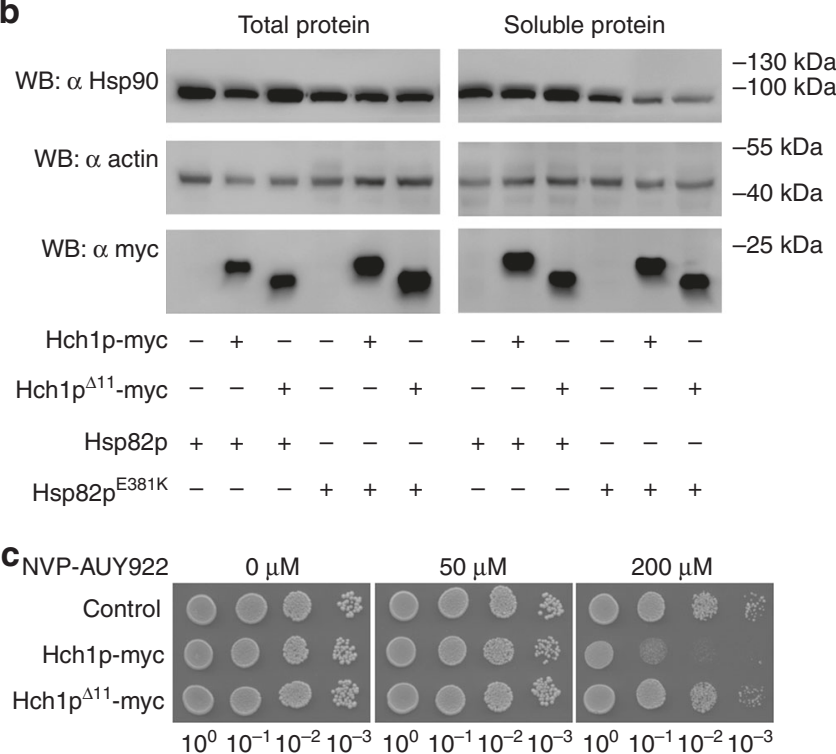

d

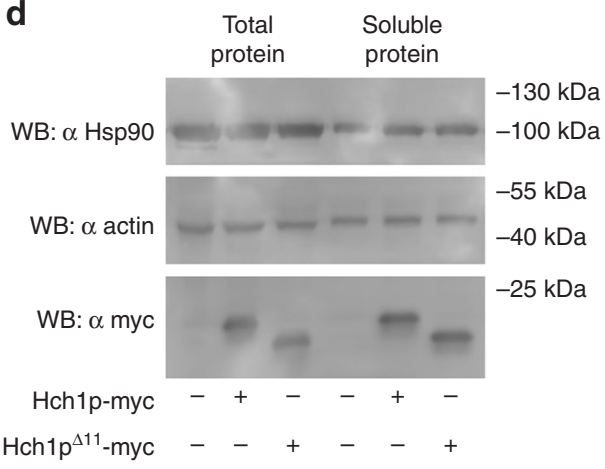

as the sole source of Hsp90 exhibit temperature-sensitive growth that is worsened by deletion of AHA1 (Fig. 5a). We used this strain to investigate Ahalp action in the context of the NxNNWHW motif. Overexpression of Ahalp in this strain rescued growth at elevated temperatures (Fig. 5b). However, overexpression of Ahalp $\mathrm{p}^{\Delta 11}$ did not have any effect on the growth of this strain. Of note, both the wildtype and $\mathrm{N}$ terminally truncated versions of Aha1p were expressed to comparable levels and were soluble (Fig. 5c). This suggests that the NxNNWHW motif is important for Ahalp function in this strain background.

The S25P mutation has not been studied before so we wondered if this substitution impaired client activation in cells. We used v-src as a marker for Hsp90 client activation with and without overexpression of Ahalp constructs. We employed a system where v-src was expressed from a galactose-inducible promoter and monitored the activation of this kinase by western blotting for the stability of $\mathrm{v}$-src as well as the accumulation of
Fig. 4 The NxNNWHW motif is required for Hch1p function in vivo. a Overexpression of myc-tagged Hch1p, but not Hch1p ${ }^{111}$, rescues growth defects of yeast expressing Hsp82pE381K. Yeast expressing wildtype Hsp82p (ip82a) or Hsp82p ${ }^{E 381 K}$ (iE381Ka) and harboring expression plasmids encoding the indicated co-chaperones were grown overnight at $30^{\circ} \mathrm{C}$ in SC media lacking uracil (SC-Ura) and containing $2 \%$ raffinose and then diluted to $1 \times 10^{8}$ cells per milliliter. We prepared 10 -fold serial dilutions and spotted $10 \mu \mathrm{L}$ aliquots on SC-Ura agar plates supplemented with either $2 \%$ glucose or galactose. Plates were incubated for 2 or 3 days for ip82a and iE381Ka strains, respectively, at $30^{\circ} \mathrm{C}$. b Western blot of total lysates and the soluble protein fraction from the yeast strains shown in a were probed with anti-Hsp90, anti-actin, and anti-myc antibodies. c Overexpression of myc-tagged Hch1p, but not Hch1p ${ }^{\Delta 11}$, confers hypersensitivity to NVP-AUY922 in yeast. Yeast expressing wildtype Hsp82p (ip82a) and harboring expression plasmids encoding the indicated co-chaperones were grown overnight at $30^{\circ} \mathrm{C}$ in YPD supplemented with $200 \mathrm{mg} / \mathrm{L} \mathrm{G} 418$ and then diluted to $1 \times 10^{8}$ cells per milliliter. We prepared 10 -fold serial dilutions and spotted $10 \mu \mathrm{L}$ aliquots on YPD agar plates supplemented with $200 \mathrm{mg} / \mathrm{L} \mathrm{G} 418$ and the indicated concentrations of NVP-AUY922. Plates were incubated for 2 days at $30^{\circ} \mathrm{C}$. d Western blot of total lysates and the soluble protein fraction from the yeast strains shown in c were probed with anti-Hsp90, anti-actin, and anti-myc antibodies. Representative results of three independent experiments are shown

phospho-tyrosine. Consistent with what we observed in our growth assays, the overexpression of Aha1p, but not Ahalp $\mathrm{p}^{\Delta 11}$, in the Hsc $82 \mathrm{p}^{\mathrm{S} 25 \mathrm{P}}$ background resulted in the accumulation of phospho-tyrosine when v-src expression was induced (Fig. 5d). Interestingly, $\mathrm{v}$-src accumulated to a comparable degree regardless of Ahalp or Ahalp $\mathrm{p}^{\Delta 11}$ expression but tyrosine kinase activity was only restored when Ahalp was overexpressed.

The S25P mutation impairs ATPase stimulation by Aha1p. We wondered if the S25P mutation conferred an ATPase defect to Hsp82p. We expressed and purified Hsp82 $\mathrm{p}^{\text {S25P }}$ for analysis in our ATPase assays. The intrinsic ATPase activity of Hsp82 $\mathrm{p}^{\mathrm{S} 25 \mathrm{P}}$ was comparable to that of wildtype Hsp82p (Fig. 6a). In fact, Hsp $82 \mathrm{p}^{\mathrm{S} 25 \mathrm{P}}$ displayed a small, but statistically significant, increase in intrinsic ATPase activity compared to wildtype Hsp82p. However, stimulation of Hsp82 $\mathrm{p}^{\mathrm{S} 2 \mathrm{P}}$ by Ahalp was vastly reduced compared to wildtype Hsp82p and was almost completely dependent on the presence of the NxNNWHW motif as the Ahalp ${ }^{\Delta 11}$ mutant barely stimulated the ATPase rate of Hsp82p ${ }^{\text {S25P }}$ (Fig. 6b).

The NxNNWHW motif modulates apparent affinity for ATP. To better understand the underlying mechanism for the different stimulated ATPase rates we observed, we measured ATP hydrolysis at different concentrations of ATP. We carried out ATPase assays with Hsp82p on its own, as well as during stimulation by Aha1p or Hch1p, with and without the NxNNWHW motif. We used varying ATP concentrations (12.5, 25, 50, 100, 200, 400, 800, $1600 \mu \mathrm{M}$ ) and analyzed the data (see Methods) to determine the apparent $K_{\mathrm{M}}$ for ATP of Hsp90 (Supplementary Figure 1). The curve fits all had $R^{2}$ values $>0.95$ ( $>0.9$ for Hsp82p alone) giving us very high confidence in the apparent $K_{\mathrm{M}}$ values we calculated.

Consistent with previous findings, we observed no significant change in the apparent $K_{\mathrm{M}}$ for ATP Hsp82p on its own or when stimulated with Aha1 $\mathrm{p}^{13}$ (Fig. 7a). Intriguingly, Hch1p resulted in a large increase in apparent $K_{\mathrm{M}}$ for ATP ( 3.5 fold) suggesting this co-chaperone reduces the affinity for nucleotide (Fig. 7b). Deletion of the NxNNWHW motif in either Aha1p or Hch1p resulted in a significant decrease in $K_{\mathrm{M}}$ relative to their full-length counterparts. 
a

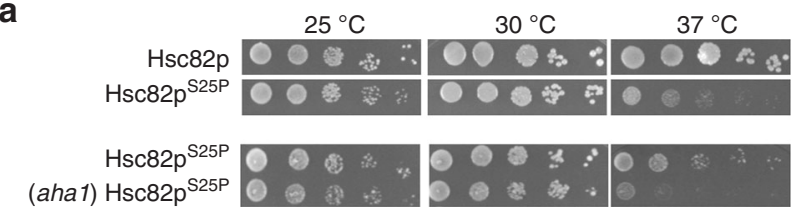

b

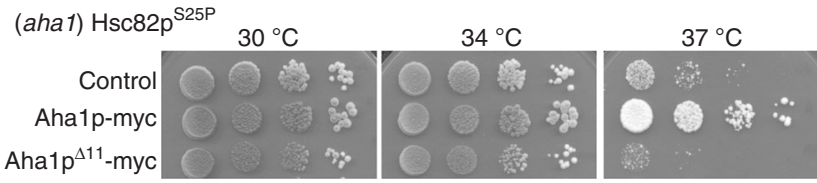

C
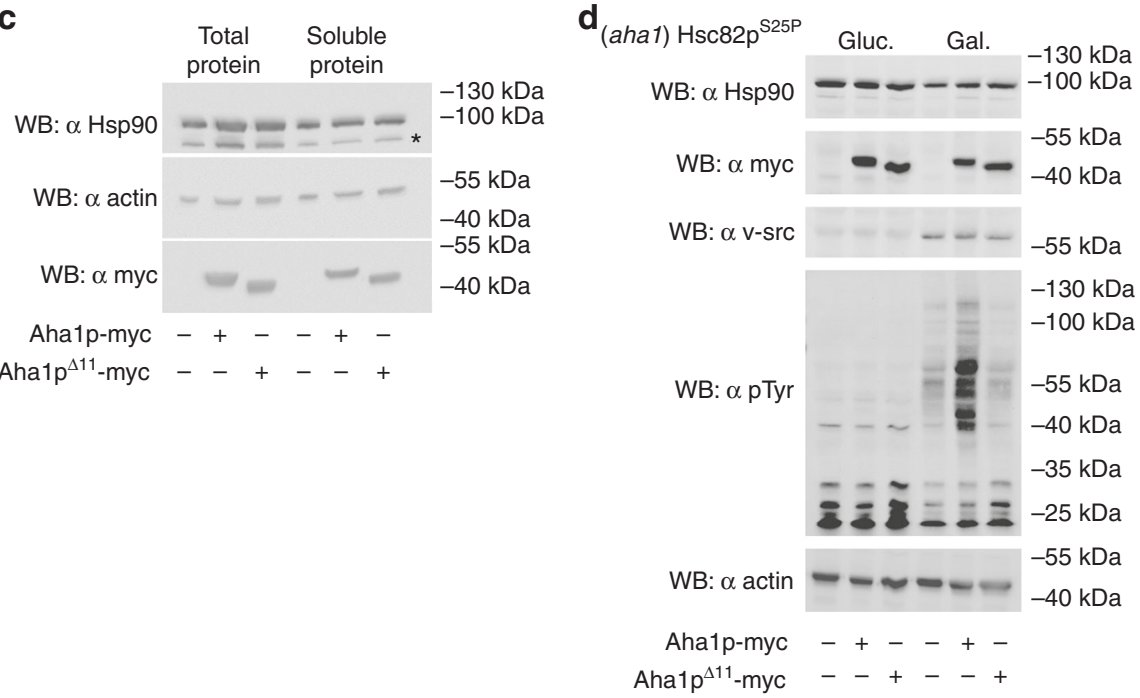

Fig. 5 The NxNNWHW motif is required for Aha1p function in vivo. a Yeast expressing Hsc82p $525 \mathrm{P}$ as the sole source of Hsp90 in the cell exhibit temperature-sensitive growth. The deletion of $\mathrm{AHA} 1$ in yeast expressing $\mathrm{Hsc} 82 \mathrm{p} \mathrm{p}^{\mathrm{S} 25 \mathrm{P}}$ exacerbates the temperature-sensitive growth defect.

b Overexpression of myc-tagged Aha1p, but not Aha1 $\mathrm{p}^{\Delta 11}$, rescues the growth of yeast expressing Hsc82 $\mathrm{p}^{\mathrm{S25P}}$. Yeast expressing Hsc82 $\mathrm{p}^{\mathrm{S25P}}$ and harboring expression plasmids encoding the indicated co-chaperones were grown overnight at $30^{\circ} \mathrm{C}$ in YPD supplemented with $300 \mathrm{mg} / \mathrm{L} \mathrm{Hygromycin}$ and then diluted to $1 \times 10^{8}$ cells per milliliter. We prepared 10 -fold serial dilutions and spotted $10 \mu \mathrm{L}$ aliquots on YPD agar plates supplemented with Hygromycin $300 \mathrm{mg} / \mathrm{L}$. Plates were incubated for 2 days at 30,34 , or $37^{\circ} \mathrm{C}$. c Western blot of total lysates and soluble protein extracted from the yeast strains shown in $\mathbf{b}$ were probed with anti-Hsp90, anti-actin, and anti-myc antibodies. d Overexpression of Aha1p, but not Aha1p ${ }^{\Delta 11}$, enhances v-src activation in yeast expressing $\mathrm{Hsc} 82 \mathrm{p}^{\mathrm{S} 25 \mathrm{P}}$. Yeast expressing $\mathrm{Hsc} 82 \mathrm{p}^{\mathrm{S25P}}$ and harboring expression plasmids encoding the indicated co-chaperones and a galactose inducible v-src expression plasmid were grown overnight at $30^{\circ} \mathrm{C}$ in SC-Ura containing $2 \%$ raffinose, supplemented with $300 \mathrm{mg} / \mathrm{L}$ hygromycin. Cells were diluted to an $\mathrm{OD}_{600}$ of 0.5 and grown for an additional $6 \mathrm{~h}$ in SC-Ura containing either $2 \%$ glucose or galactose, supplemented with hygromycin (300 mg/L). Yeast strains were probed with anti-Hsp90, anti-myc, anti-v-src, anti-phosphotyrosine, and anti-actin antibodies. Representative results of three independent experiments are shown

The NxNNWHW motif does not influence lid closure. ATP hydrolysis by $\mathrm{Hsp} 90$ is rate-limited by conformational change ${ }^{34,45,46}$. Local conformational changes within the chaperone machinery are kinetically linked and appear to cooperate ${ }^{34}$. Briefly, ATP binding drives closure of the lid over the binding pocket, exchange of $\mathrm{N}$ terminal strands between the two protomers of the $\mathrm{N}$ terminally dimerized $\mathrm{Hsp} 90$, and docking of the $\mathrm{N}$ domains with the middle domains of Hsp90. These coordinated events are stimulated when Ahalp binds to Hsp90 34 . Following the structural model (Fig. 2b-d), the NxNNWHW-containing peptide of Ahalp is located in close proximity to the $\mathrm{N}$ domain of Hsp90. Presence of this conserved motif is thus likely to influence the $\mathrm{N}$ terminal ATP-binding domain of Hsp90, which appears to be reflected in our observed alteration of $K_{\mathrm{M}}$. To explore the mechanistic basis for reduced ATPase stimulation of Hsp90 by Ahalp $\mathrm{p}^{\Delta 11}$ and the associated reduction of $K_{\mathrm{M}}$ of ATP we measured the rate constants of closure of the lid over the ATPbinding pocket in the $\mathrm{N}$ domain using photoinduced electron transfer (PET) fluorescence quenching, as previously described ${ }^{34}$.
Previous work demonstrated that Ahalp accelerates closure of the lid and the other associated conformational events. In Michaelis-Menten kinetics, a reduction of $K_{M}$ can originate from a reduction of rate constant of ATP hydrolysis or from modulation of ATP binding (i.e. from a reduced rate constant of ATP dissociation or increased rate constant of ATP association), or both $^{47,48}$. We hypothesized that the deletion of the NxNNWHW motif would likely affect kinetics of conformational change (i.e. would slow lid closure and therefore reduce the rate constant of ATP hydrolysis by Hsp90). We measured the time constant $(\tau)$ of lid closure of yeast Hsp90, which was $\tau=320 \pm 100 \mathrm{~s}$ (Fig. 8a), in agreement with previous findings ${ }^{34}$. Surprisingly, we found that lid closure was accelerated to an identical degree by Ahalp and Ahalp $\mathrm{p}^{\Delta 11}(\tau=14 \pm 2 \mathrm{~s}$ and $\tau=15 \pm 2 \mathrm{~s}$, respectively), showing that the decrease in ATPase stimulation we observed with Ahalp $\mathrm{p}^{\Delta 11}$ was not due to a defect in the acquisition of the catalytically competent state (Fig. 8b). We next wondered how Hchlp (which regulates Hsp90 differently in cells ${ }^{7,30}$ ) affects kinetics of lid closure and acquisition of the catalytically 

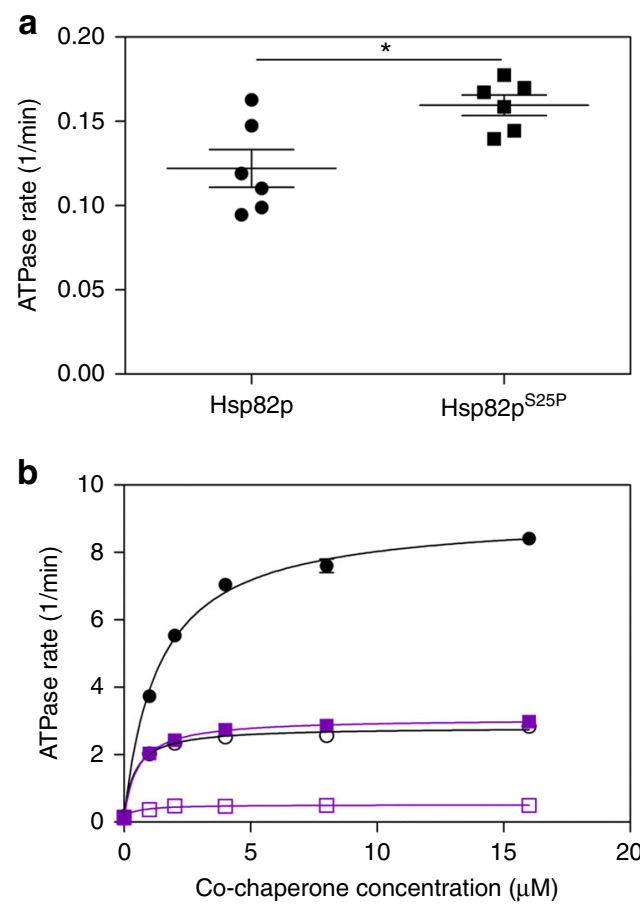

Fig. $6 \mathrm{Hsp} 82 \mathrm{p}^{\mathrm{S25P}}$ ATPase stimulation by Aha1p is dependent on the NxNNWHW motif. a The intrinsic ATPase activity of Hsp82p $525 \mathrm{P}$ is comparable to that of wildtype Hsp82p. Reactions contained $5 \mu \mathrm{M} \mathrm{Hsp82p}$ or Hsp82p ${ }^{\mathrm{S} 25 \mathrm{P}}(n=6)$. Statistical significance $\left({ }^{\star}\right)$ was determined using an unpaired $t$-test. Error bars show standard error of the mean. $\mathbf{b}$ Stimulation of the ATPase activity of wildtype Hsp82p and Hsp82p 25 P by increasing concentrations of Aha1p (closed black circles, open black circles, respectively) and $A h a 1 p^{\Delta 11}$ (closed purple squares, open purple squares, respectively). Reactions contained $1 \mu \mathrm{M} \mathrm{Hsp82p}$ and indicated concentration of co-chaperone $(n=3)$. Error bars show standard error of the mean

competent state. We tested Hch1p and Hch $1 \mathrm{p}^{\Delta 11}$ in this assay and found, again, that time constants of lid closure were within error, i.e. $(\tau=105 \pm 13 \mathrm{~s}$ and $\tau=120 \pm 16 \mathrm{~s}$, respectively) (Fig. 8c). Unchanged kinetics of conformational change lead us to conclude that reduction of $K_{\mathrm{M}}$ of ATP/Hsp90 in Aha-type NxNNWHW deletion mutants compared with wildtype Ahal originated from tighter binding of nucleotide in the $\mathrm{N}$ domainbinding pocket (i.e. from a reduced dissociation constant of bound nucleotide induced by interaction with the NxNNWHW motif). Interestingly, binding of Aha-type co-chaperones with and without the NxNNWHW motif resulted in different fluorescence intensities of the label positioned on the $\mathrm{N}$ terminal domain (Fig. 8b, c), which shows that there is indeed an interaction of the NxNNWHW motif with the $\mathrm{N}$ terminal domain.

Loss of the NxNNWHW motif promotes the Hsp90 closed state. Loss of the NXNNWHW motif did not appear to slow the acquisition of the catalytically competent state of Hsp90 compared to full-length Ahalp in our PET fluorescence measurements. This was surprising since ATPase stimulation of Hsp90 by Ahalp ${ }^{\Delta 11}$ was impaired at steady state. We speculated that this could be because the NxNNWHW is involved in release of ADP after hydrolysis has occurred. Slower ADP release would result in a slower cycling rate. Sbalp is a co-chaperone that binds to the $\mathrm{N}$ terminally dimerized, catalytically competent state of $\mathrm{Hsp} 90^{33}$. Sba1p does not affect Hsp90 ATPase activity in single turnover reactions but inhibits ATPase activity at steady state ${ }^{49}$. This is because it slows the release of ADP after hydrolysis has occurred.

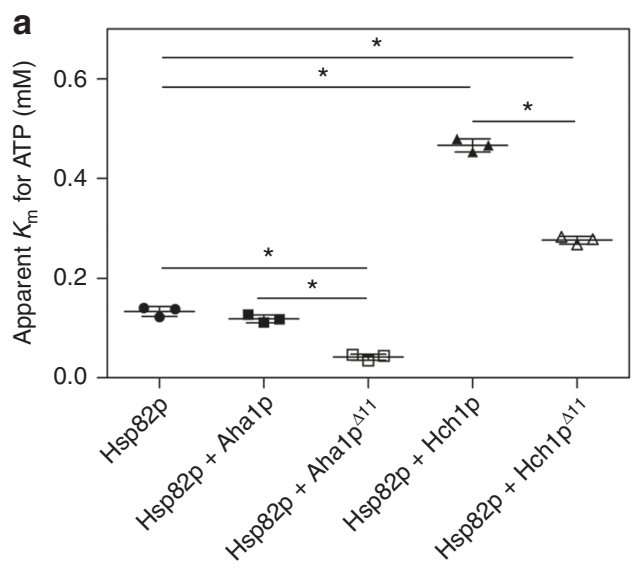

b

\begin{tabular}{|l|l|}
\hline Condition & Apparent $K_{\mathrm{m}}$ for ATP \\
\hline Hsp82p alone & $0.133+/-0.010$ \\
\hline Hsp82p + Aha1p & $0.118+/-0.008$ \\
\hline Hsp82p + Aha1 ${ }^{\Delta 11}$ & $0.042+/-0.006$ \\
\hline Hsp82p + Hch1p & $0.466+/-0.013$ \\
\hline Hsp82p + Hch1p ${ }^{\Delta 11}$ & $0.277+/-0.008$ \\
\hline
\end{tabular}

Fig. 7 The NxNNWHW motif modulates the apparent $K_{M}$ for ATP of Hsp90. a Kinetic analysis was carried out for Hsp82p alone or in the presence of Aha1p, Aha1p ${ }^{\Delta 11}$, Hch1p, or Hch1p ${ }^{\Delta 11}$. ATPase reactions were carried out with increasing concentrations of ATP $(12.5,25,50,100,200$, $400,800,1600 \mu \mathrm{M})$ and ATPase rates were analyzed with the Michaelis-Menten non-linear regression function in GraphPad Prism. The curve fits all had $R^{2}$ values $>0.9$. The apparent $K_{\mathrm{M}}$ values for three experiments are shown in the scatter plots. Statistical significance $\left({ }^{*}\right)$ was calculated pair-wise with a $t$-test $(n=3 ; n=4$ for Hsp82p alone). Error bars show standard deviation. $\mathbf{b}$ Table showing $K_{\mathrm{M}}$ values plotted in a

If Ahalp ${ }^{\Delta 11}$ promotes the acquisition of the ATPase-competent state but cannot promote ADP dissociation then we would predict the apparent affinity of Sbalp would be higher for Hsp90 in the presence of Ahalp $\mathrm{p}^{\Delta 11}$ than in the presence of Ahalp. To test this, we titrated Sbalp into Hsp90 ATPase reactions stimulated with either Ahalp or Ahalp ${ }^{\Delta 11}$. Strikingly, despite Ahalp $\mathrm{p}^{\Delta 11}$ having a higher apparent affinity for Hsp82p than Aha1p, Sbalp had a far higher apparent affinity for Hsp82p that was stimulated by Aha1p $\mathrm{p}^{\Delta 11}$ (Fig. 9).

\section{Discussion}

Ahalp and Hchlp share several sequence elements that are important for regulating the ATPase activity of $\mathrm{Hsp} 90$ in vitro as well as Hsp90 function in vivo $7,13,19,30,44$. We report here that the strongly conserved NxNNWHW motif, harbored in the first 11 amino acids of Ahalp and Hchlp (and all Aha-type co-chaperones) (Fig. 1), is critical for the in vivo function of both co-chaperones. This region is not fully resolved in the co-crystal structure of the Hsp90 middle domain and the Ahalp $\mathrm{N}$ domain ${ }^{19}$. However, its location can be inferred from the location and orientation of the Ahalp $\mathrm{N}$ domain in complex with the Hsp90 middle domain ${ }^{19}$ in the context of the structure of full-length $\mathrm{Hsp} 90^{33,35,36}$. The NxNNWHW motif extends towards the $\mathrm{N}$ terminal Hsp90 ATPase domains (Fig. 2). This is consistent with our enzymatic data which shows that the NxNNWHW motif is important for ATPase stimulation by both co-chaperones (Fig. 3).

Several intra-subunit and inter-subunit rearrangements occur during the Hsp90 ATP hydrolysis cycle. Access to the nucleotidebinding pocket is controlled by a lid (amino acids 98-121) ${ }^{50}$. This lid is in the open position in the absence of nucleotide but 

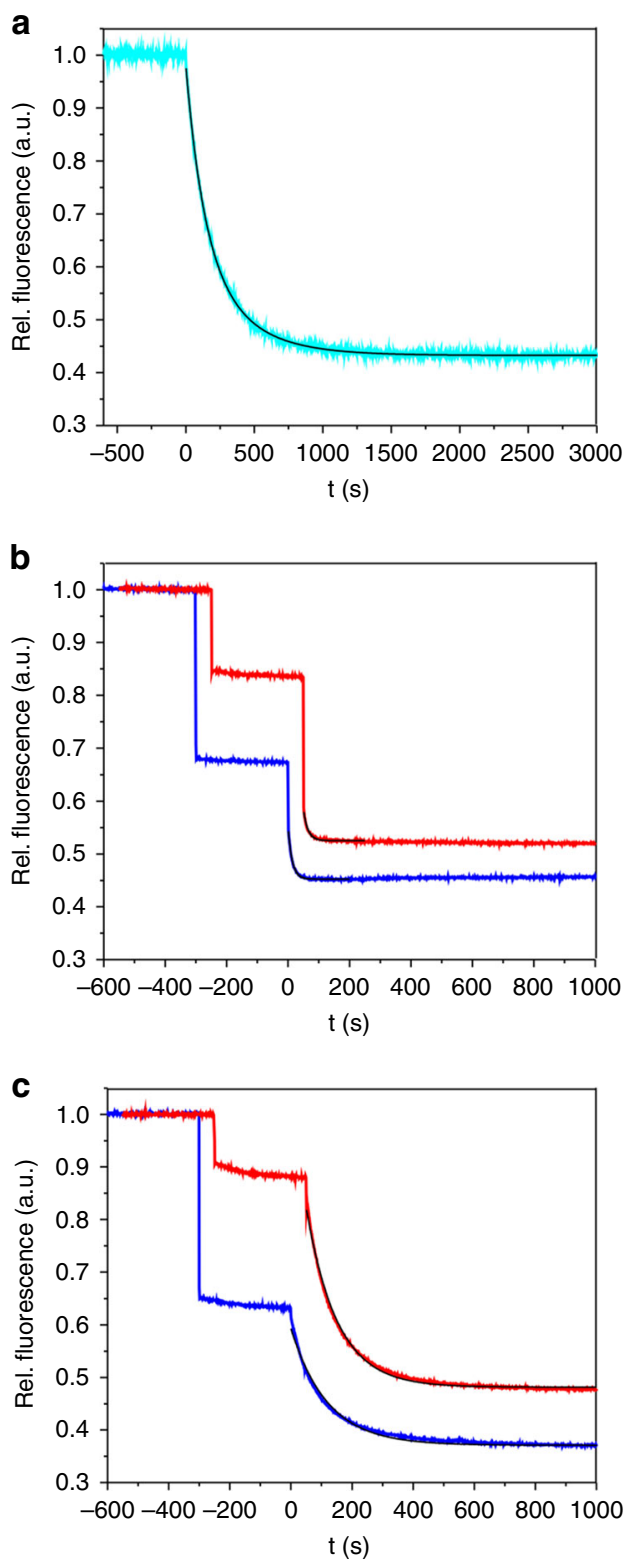

Fig. 8 Kinetics of lid closure of Hsp90 and its modulation by Aha-type cochaperones. a Time course of PET fluorescence quenching of fluorescently modified Hsp90 mutant S51C-A110W (cyan). The non-hydrolyzable ATP analog AMP-PNP, which traps Hsp90 in the closed-clamp conformation, was added at time $t=0$. The black line is a fit to a bi-exponential decay function. $\mathbf{b}$ Same experiment as shown in a but Ahalp (blue line) or Aha1p ${ }^{\Delta 11}$ (red line) was added 300 s prior to addition of AMP-PNP. Black lines are fits to exponential decay functions. c Same experiment as shown in a but Hch1p (blue line) or Hch1p ${ }^{\Delta 11}$ (red line) was added $300 \mathrm{~s}$ prior to addition of AMP-PNP. Black lines are fits to exponential decay functions. Data sets of Aha1p $\mathrm{p}^{\Delta 11}$ and of Hch1p $\mathrm{p}^{\Delta 11}$ are offset along the $\mathrm{x}$-axis for reasons of clarity

undergoes a rapid remodeling event followed by slow closure upon ATP binding ${ }^{34}$. This traps ATP in the binding pocket and renders it committed to hydrolysis ${ }^{51}$. Lid closure is accompanied by dimerization of the $\mathrm{N}$ terminal domains and exchange of a beta strand (amino acids 1-8) between the two protomers ${ }^{9,34,46,50,52}$. This conformation is thought to be catalytically active. The conformational changes that occur after ATPbinding comprise the rate limiting step(s) of the ATPase cycle ${ }^{51}$. Ahalp is well known to activate Hsp90 ATPase activity by

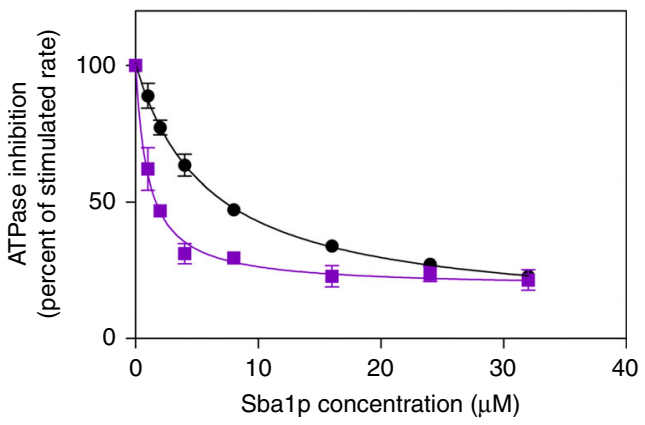

Fig. 9 Sba1 has a higher apparent affinity for Hsp82p in the presence of Aha1p ${ }^{\Delta 11}$. Increasing concentrations of Sba1p were titrated into reactions containing Hsp82p and either Aha1p (black circles) or Aha1p ${ }^{\Delta 11}$ (purple squares) $(n=4)$. Reactions contained $1 \mu \mathrm{M} \mathrm{Hsp82p,} 5 \mu \mathrm{M}$ of either Aha1p or Aha1p ${ }^{\Delta 11}$, and the indicated concentration of Sba1p. Error bars show standard deviation

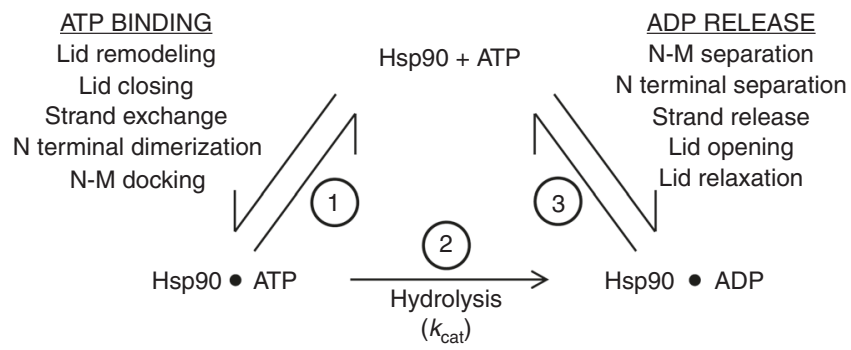

Fig. 10 A simplified schematic of the Hsp90 ATPase cycle. ATP binding is accompanied by several conformational rearrangements that must occur for Hsp90 to attain its catalytic state (1). Once ATP hydrolysis occurs (2), these conformational changes must be reversed for ADP to be released and another round of ATP binding and hydrolysis to be initiated (3)

accelerating these steps $9,34,46,53,54$. After ATP hydrolysis occurs, the molecular clamp must open in order for ADP and inorganic phosphate to be released and a new cycle of ATP binding and hydrolysis to be initiated (Fig. 10). However, the order and kinetics of events associated with ADP release after ATP hydrolysis are not well understood, especially in the context of stimulation by co-chaperones-like Ahalp and Hchlp, and are extremely difficult to measure directly.

Deletion of the NxNNWHW motif results in a clear defect in ATPase stimulation of Hsp90 (Fig. 3). A defect in the acquisition of the catalytic state seems like the simplest explanation for the reduction in $V_{\max }$ we observe with Ahalp ${ }^{\Delta 11}$. Such a defect could be in the ability of Ahalp to mobilize the catalytic loop when the NxNNWHW motif is deleted. However, deletion of the NxNNWHW motif did not impair Hsp90 ATPase stimulation by the Ahalp $\mathrm{N}$ terminal domain on its own (Fig. 3d) suggesting that both Ahal ${ }^{\mathrm{N}}$ and Ahal ${ }^{\mathrm{N}-\Delta 11}$ are equally able to remodel the catalytic loop. The ATPase stimulation defect we observe upon deletion of the NxNNWHW motif in full length Ahalp appears to be dependent on the presence of the Ahalp $\mathrm{C}$ terminal domain (Fig. 3b). Thus, in Ahalp, the NxNNWHW motif and the C terminal domain act cooperatively to promote full stimulation of Hsp90 at steady state. The $\mathrm{C}$ domain of Ahalp is required for robust ATPase stimulation (compared to the Ahalp $\mathrm{N}$ domain alone) not because of a role in catalytic loop remodeling, but rather because of its interaction with the Hsp90 $\mathrm{N}$ domains and acquisition of the $\mathrm{N}$ terminally dimerized state ${ }^{35-37}$. However, cochaperone switching (Stilp displacement) was equally efficient with either Ahalp $\mathrm{p}^{\Delta 11}$ or Ahalp (Fig. 3e). In addition, Sbalp, which 
is a sensor for the $\mathrm{N}$ terminally dimerized state of $\mathrm{Hsp} 90^{55,56 \text {, }}$ actually bound with a higher apparent affinity to Hsp90 in complex with Ahalp $\mathrm{p}^{\Delta 11}$ than with Ahalp (Fig. 9). This observation may provide the strongest clue regarding the role of Ahalp in nucleotide release because Sbalp does not inhibit ATPase hydrolysis in single turnover reactions but robustly inhibits at steady state ${ }^{49}$. Sbalp stabilizes the $\mathrm{N}$ terminally dimerized state of Hsp90 and slows ADP release, after which Sbalp can no longer bind. A higher apparent Sbalp-binding affinity is consistent with ADP release being slower in the presence of Ahalp ${ }^{\Delta 11}$ than wildtype Ahalp. Of course we must acknowledge that the NxNNWHW motif may pose a steric barrier to Sbalp binding as well. In all these experiments we were unable to identify a defect in the ability of the Ahalp ${ }^{\Delta 11}$ mutant to promote the catalytically active, $\mathrm{N}$ terminally dimerized state. In fact, direct measurement of lid closure revealed identical rates with Ahalp and Ahalp ${ }^{\Delta 11}$ (Fig. 8b). What then could account for the reduction in $V_{\max }$ in our cycling ATPase assays with Ahalp ${ }^{\Delta 11}$ ? We observed an increase in apparent affinity for nucleotide in ATPase assays with both Ahalp $\mathrm{p}^{\Delta 11}$ and Hchlp $\mathrm{p}^{\Delta 11}$ compared to their full length counterparts (Fig. 7). Since $k_{\text {on }}$ is diffusion controlled and closing of the clamp was unaltered by the deletion of NxNNWHW motif, we propose that the rate of ADP release (after hydrolysis) is slower in the presence of Ahalp $\mathrm{p}^{\Delta 11}$ compared to Ahalp. Certainly, dissociation of ADP would be rate limiting for a new cycle of ATP hydrolysis to be initiated at steady state (Fig. 10).

Deletion of the NxNNWHW motif affects Ahalp and Hchlp in a similar manner despite the different roles these cochaperones play in regulating Hsp90 biology in yeast ${ }^{7,30}$. Ahalp is known to elicit a partially closed lid conformation that one would expect to make ATP-trapping more efficient and lower the $K_{\mathrm{M}}$ for $\mathrm{ATP}^{5,9,46}$. However, in agreement with previous studies, we did not observe a change in the apparent $K_{M}$ for ATP in Ahalp-stimulated reactions compared to reactions containing Hsp90 alone ${ }^{13}$. There was no difference between stimulated ATPase rates in reactions containing the Ahalp $\mathrm{N}$ domain or a variant lacking the NxNNWHW motif in our hands (Fig. 3d). This suggests that, at least for Ahalp, the NxNNWHW motif acts cooperatively with the $\mathrm{C}$ domain. In the case of Hchlp, the pronounced effect on the apparent $K_{\mathrm{M}}$ of Hsp90 for ATP compared to Ahalp may explain the ability of Hchlp to regulate cellular sensitivity to Hsp90 inhibitors ${ }^{7}$. Overexpression of Hchlp, but not Ahalp, confers sensitivity to Hsp90 inhibitors in yeast ${ }^{7}$. If Hchlp stimulates Hsp90 ATPase activity in part by promoting nucleotide exchange, this may allow greater access for ATP-competitive Hsp90 inhibitors to the ATP-binding pocket. The deletion of the NxNNWHW motif in Hch1p dramatically lowered the ATPase rate and $K_{\mathrm{M}}$ for ATP of Hsp90 and ablated the ability of Hchlp to sensitize yeast to Hsp90 inhibitors.

Deletion of the NxNNWHW in either Hchlp or Ahalp eliminates the in vivo activity of these co-chaperones while only affecting nucleotide exchange in vitro. That the deletion of the NxNNWHW motif does not affect the ability of Ahalp $p^{\Delta 11}$ and $\mathrm{Hch}_{1} \mathrm{p}^{\Delta 11}$ to promote the acquisition of the catalytically active state but eliminates their biological activity suggests that nucleotide exchange (or release) is the critical function of these co-chaperones. The precise manner in which ATP hydrolysis and nucleotide exchange fit into the client activation cycle has not been elucidated. Recent work has even called the significance of ATP hydrolysis into question while reaffirming the importance of nucleotide binding for Hsp90 function ${ }^{39}$. Additionally, there is also some evidence that mixed nucleotidebound states (one protomer bound to ADP and one bound to ATP) may also be functionally significant ${ }^{57}$. Focusing on other elements of the functional cycle, such as exchange dynamics during cycling, may reveal new insight into how client activation is regulated.

\section{Methods}

Yeast strains and plasmids. Yeast galactose-inducible plasmids were constructed by amplifying $H C H 1$ and $A H A 1$ coding sequences (wildtype (primer 13 for AHA1; primer 17 for $\mathrm{HCH} 1$ ) or with the 11 amino acids harboring the NxNNWHW motif deleted $(\triangle 11)$ (primer 573 for $A H A 1$; primer 571 for $H C H 1)$ ) by PCR to have upstream BamHI and downstream SacI (primer 90 for HCH1) or NotI (primer 126 for AHA1) sites for cloning into pRS416GAL (for wildtype Aha1p and Hch1p) or pRS426GAL (for Ahalp ${ }^{\Delta 11}$ and Hchlp $\left.{ }^{\Delta 11}\right)^{58}$. The HCH1 and $A H A 1$ coding sequences (wildtype (primer 13 for $A H A 1$; primer 17 for $H C H 1$ ) or $\triangle 11$ variant (primer 573 for $A H A 1$; primer 571 for $H C H 1)$ ) were amplified by PCR with primers designed to introduce a BamHI site at the $5^{\prime}$ end and a myc tag and XhoI site at the $3^{\prime}$ end (primer 16 for AHA1; primer 20 for $H C H 1$ ). These PCR products were then digested with BamHI and XhoI and cloned into similarly cut p41KanTEF ${ }^{7}$. Site-directed mutagenesis for Hsp82(S25P) was carried out using QuikChange ${ }^{\mathrm{Tm}}$ mutagenesis according to the manufacturers protocol (Agilent) using primers 672 and 673. The coding sequences contained in all mutagenized plasmids were verified by sequencing. We constructed our p42HygGPD vector by digesting the GPD and CYC1 terminator fragment of p414GPD ${ }^{59}$ with SacI and KpnI and ligating into similarly cut pRS42 $\mathrm{H}^{60}$. The $H C H 1$ and $A H A 1$ (wildtype or $\triangle 11$ variant) coding sequences were cut from p41KanTEF plasmids using BamHI and XhoI and cloned into p42HgyGPD cut BamHI and SalI to yield p42HygGPDHch1myc, p42HygGPDHch $1^{\Delta 11}$ myc, p42HygGPDAhalmyc, and p42HygGPDAhal ${ }^{\Delta 11}$ myc. The yeast strains ip82a, $\mathrm{iE} 381 \mathrm{~K}$, and iTHisHsp82p were derived from $\triangle$ PCLDa (kindly provided by Dr. Susan Lindquist $)^{7,30,61}$. In the case of yeast strains transformed with our p41KanTEF or p42HygGPD plasmids, transformants were selected on YPD supplemented with G418 (200 mg/L) (Goldbio, USA) or Hygromycin $(300 \mathrm{mg} / \mathrm{L}$ ) (Goldbio, USA) respectively.

Bacterial expression vectors encoding $\mathrm{N}$ terminally His-tagged Hch1p and Ahalp lacking the first 11 amino acids $(\Delta 11)$ were constructed in a similar manner to those encoding wildtype Ahalp and Hchlp $7,30,37$. We amplified the $\mathrm{HCH} 1$ (primers 601/27) and AHA1 (primer 572/29) coding sequences were amplified by PCR to introduce an NdeI site at the $5^{\prime}$ end and a BamHI site at the $3^{\prime}$ end. These products were then digested with $\mathrm{NdeI}$ and $\mathrm{BamHI}$ and ligated into similarly cut pET11dHis. Bacterial expression vectors encoding C terminally His-tagged Hchlp and Ahalp (wildtype (primers 657/659 for AHA1; primer 654/656 for $\mathrm{HCH}$ ) and lacking the first 11 amino acids $(\Delta 11)$ (primer 658/659 for $A H A 1$; primer 655/656 for $H C H 1)$ ) were constructed by amplifying $H C H 1$ and $A H A 1$ coding sequences by PCR to introduce an $\mathrm{NcoI}(\mathrm{HCH} 1)$ or $\mathrm{XbaI}$ (AHA1) site at the $5^{\prime}$ and a BamHI site at the $3^{\prime}$ end. These PCR products were digested with NcoI and BamHI (HCH1) or XbaI and BamHI (AHA1) and ligated into similarly cut pET11dHis. For C-terminally His-tagged Aha1 $\mathrm{p}^{\mathrm{N}}$ domain, the same procedure was followed as for Ahalp except primers 657 and 664 were used (primers 658 and 664 for the $\Delta 11$ variant). All vectors were sequenced to verify final coding sequences.

The Hsc82 $\mathrm{p}^{\mathrm{S} 25 \mathrm{P}}$ mutant was identified in a yeast screen ${ }^{62}$. Briefly, the coding sequence of HSC 82 was subjected to error-prone mutagenesis. The library was screened by transformation into an $h s c 82 h s p 82$ strain or $h s c 82 h s p 82$ strain containing deletion of non-essential co-chaperones and selecting colonies that failed to support growth when plated onto 5-FOA at $37^{\circ} \mathrm{C}$. Library plasmids that produced wildtype levels of Hsc82p were rescued and sequenced fully. The pRS316 v-src plasmid was a gift from Dr. David Morgan (University of California) ${ }^{63}$ and transformants were selected on SC-uracil. All primers are listed in Supplementary Figure 2.

Growth assays. Strains were grown in defined media or YPD (where indicated) with or without G418 $(200 \mathrm{mg} / \mathrm{L})$ or hygromycin $(300 \mathrm{mg} / \mathrm{L})$, diluted to $1 \times 10^{8}$ cells per $\mathrm{mL}$ and 10 -fold serial dilutions were prepared as indicated. Ten microliters of drops were placed on agar plates (YPD or defined, with or without NVPAUY922 (at indicated concentrations), with or without G418 or hygromycin) and grown for $48 \mathrm{~h}$ unless otherwise indicated, at indicated temperatures. Growth assays with yeast expressing $\mathrm{Hsc} 82 \mathrm{p}^{\mathrm{S} 25 \mathrm{P}}$ were carried out as above in strain JJ816 (hsc82hsp82) and JJ95 (aha1hsc82hsp82) ${ }^{64}$.

v-src activation assay. Yeast were grown overnight at $30^{\circ} \mathrm{C}$ in SC-Ura with $2 \%$ raffinose. After overnight growth, cells were diluted to an $\mathrm{OD}_{600}$ of 0.5 and grown for an additional $6 \mathrm{~h}$ rotating at $30^{\circ} \mathrm{C}$ in $5 \mathrm{~mL}$ of appropriate media supplemented with $2 \%$ glucose, for inhibition of the plasmid, or $2 \%$ galactose, for plasmid induction. After $6 \mathrm{~h}$ the $\mathrm{OD}_{600}$ was measured and five units of cells were harvested for protein extraction and analyzed by western blot.

Lysate generation and Western blotting. Yeast were grown overnight at $30^{\circ} \mathrm{C}$ in appropriate media. For analysis of total protein content, five $\mathrm{OD}_{600}$ units of cells were transferred to a microfuge tube, washed with distilled water and then pelleted for processing. Cells were resuspended in $500 \mu \mathrm{L}$ of distilled water, $90 \mu \mathrm{L}$ of lysis buffer (2.2 M NaOH, $1 \mathrm{M} \beta$-mercaptoethanol, $10 \mathrm{mM}$ PMSF) was added and samples vortexed twice for 30 s. $250 \mu \mathrm{L}$ of $100 \%$ TCA was added and samples were vortexed briefly and then precipitated in a cold microcentrifuge. Pellets were washed twice with acetone, dried and then resuspended in sample buffer for analysis by SDS-PAGE and western blotting. For analysis of soluble protein content, $35 \mathrm{OD}_{600}$ units of cells were resuspended in $1 \mathrm{~mL}$ of lysis buffer $(50 \mathrm{mM}$ Tris 
$\mathrm{pH} 7.5,100 \mathrm{mM} \mathrm{KCl}, 5 \mathrm{mM} \mathrm{MgCl} 2,20 \mathrm{mM} \mathrm{Na}_{2} \mathrm{MoO}_{4}, 20 \%$ glycerol, $5 \mathrm{mM}$ $\beta$-mercaptoethanol, HALT EDTA-free protease inhibitor (Thermo scientific)) and added to a $2-\mathrm{mL}$ screw cap tube that was half-filled with $0.5 \mathrm{~mm}$ glass beads (Biospec, Bartlesville, OK, USA). Cells were lysed with a Mini-Beadbeater-16 for $3 \mathrm{~min}$, and supernatant was clarified by centrifugation at 20,800 $\mathrm{rcf}$ for $10 \mathrm{~min}$. Protein from each supernatant was TCA precipitated and resuspended in sample buffer for analysis by SDS-PAGE and western blotting. Myc-tagged proteins were detected with mouse anti-myc monoclonal antibody ${ }^{65}$ (1:100; 4A6 Millipore, catalog number 05-724) and Hsp82p was detected with anti-Hsp90 antibody (1:1000; Anti-Hsp90, Clone K41220A, Stressmarq Biosciences Inc., Victoria, BC, Canada; catalog number SMC-135), v-src was detected with anti-v-src antibody (1:200; clone 327 Sigma-Aldrich; catalog number MABS193), phosphotyrosine levels were detected with anti-phosphotyrosine antibody (1:1000; Stressmarq Biosciences Inc., Victoria, BC, Canada; catalog number SMC-157). Anti-actin antibodies were kindly provided by Dr. Gary Eitzen (1:2000; University of Alberta).

Genomic analysis. We performed reciprocal BLAST searches with the Hchlp and Ahalp protein sequences against a large number of published genomes with which predicted proteins sequences exist. Our criteria for classification of proteins as Hchlp-like or Ahalp-like are as follows. Hchlp-like proteins contained one or more of the NxNNWHW and RKxK motifs and D53 but lacked a recognizable C-terminal domain similar to Ahalp $\mathrm{p}^{157-350}$. Ahalp-like proteins contained one or more of the NxNNWHW and RKxK motifs, and D53 and possessed a recognizable C-terminal domain.

Protein expression and purification. Saccharomyces cerevisiae Hsp82p, Ahalp, Ahalp ${ }^{\Delta 11}$, Hchlp, Hchlp ${ }^{\Delta 11}$, Cpr6p, and Stilp were expressed in Escherichia coli strain BL21 (DE3) (New England Biolabs) from pET11d (Stratagene, La Jolla, CA, USA). Two versions of pET11d were used to express these proteins. Hsp82p, Ahalp, Ahal ${ }^{\Delta 11}$, Cpr6p, and Stilp were expressed with $N$ terminal $6 x H i s$ tags and Ahalp, Ahalp ${ }^{\Delta 11}$, Ahalp ${ }^{\mathrm{N}}$, Ahalp ${ }^{\mathrm{N}-\Delta 11}, \mathrm{Hch} 1 \mathrm{p}$, and Hchlp ${ }^{\Delta 11}$ were expressed with $\mathrm{C}$ terminal $6 \mathrm{xHis}$ tags. Cells were grown at $37^{\circ} \mathrm{C}$ to an $\mathrm{OD}_{600}$ of 0.8-1.0 and induced with $1 \mathrm{mM}$ isopropyl-1-thio-D-galactopyranoside (IPTG). Cells expressing Hchlp, Hchlp $p^{\Delta 11}$, Ahalp, Ahal $p^{\Delta 11}$, and Hsp82p were harvested after overnight growth at $30^{\circ} \mathrm{C}$. Cells expressing Cpr6p, and Stilp were harvested after overnight growth at $37^{\circ} \mathrm{C}$. Cells were harvested by centrifugation and stored at $-80^{\circ} \mathrm{C}$. Cells were resuspended in lysis buffer $\left(25 \mathrm{mM} \mathrm{NaH}_{2} \mathrm{PO}_{4}, \mathrm{pH} 7.2\right.$, $500 \mathrm{mM} \mathrm{NaCl}, 1 \mathrm{mM} \mathrm{MgCl}$, $20 \mathrm{mM}$ Imidazole, $5 \mathrm{mM} \beta$-mercaptoethanol) and lysed using Avestin Emulsiflex C3 (Avestin, Ottawa, Ontario, Canada). Lysates were clarified by ultracentrifugation and His-tagged proteins were isolated on a HisTrap FF column using an AKTA Explorer FPLC (GE Healthcare). Isolated 6xHis-tagged proteins were then concentrated and further purified by size exclusion chromatography on a Superdex 200 (Hsp82p, Stilp, Ahalp, Aha1p ${ }^{411}$, Hchlp, Hch1 $\mathrm{p}^{\Delta 11}$ ) or a Superdex 75 (Cpr6p) column (GE Healthcare) ${ }^{7,30}$. Purity of each protein preparation was $>95 \%$ as verified by coomassie-stained SDS-PAGE analysis.

ThermoFluor thermal shift assay. Thermal stability was used as an indicator of folding status of our C terminally 6xHis-tagged Ahalp, Ahalp ${ }^{\Delta 11}$, Hchlp, and Hchl $\mathrm{p}^{\Delta 11}$. We carried out a thermal shift assay with 5, 2, and $1 \mu \mathrm{M}$ concentrations of each co-chaperone construct in triplicate using Sypro Orange (ThermoFisher Scientific ${ }^{42,43} . T_{\mathrm{m}}$ was calculated using area under the curve analysis in GraphPad Prism of plots of $\Delta F / \Delta T$ (change in fluorescence/change in temperature).

In vitro ATPase assays. ATPase assays were carried out using the enzyme coupled assay as previously described $7,17,30,40,41$. All reactions were carried out in triplicate, three times in $100 \mu \mathrm{L}$ volumes using a 96-well plate. Absorbance at $340 \mathrm{~nm}$ was measured every minute for 90 min using a BioTek Synergy 4 and the path-length correction function. Average values of the experiments are shown with error expressed as standard error of the mean. The decrease in NADH absorbance at 340 $\mathrm{nm}$ was converted to micromoles of ATP using Beer's Law and then expressed as a function of time ${ }^{40}$. The final conditions of all the reactions are $25 \mathrm{mM}$ Hepes $(\mathrm{pH}$ 7.2), 12.5 or $16 \mathrm{mM} \mathrm{NaCl}$ (in titration and cycling experiments, respectively), 5 $\mathrm{mM} \mathrm{MgCl} 2,1 \mathrm{mM}$ DTT, $0.6 \mathrm{mM} \mathrm{NADH}, 2 \mathrm{mM} \mathrm{ATP}$ (co-chaperone titration and cycling experiments), $1 \mathrm{mM}$ phosphoenol pyruvate (PEP), $2.5 \mu \mathrm{L}$ of pyruvate kinase/lactate dehydrogenase (PK/LDH) (Sigma), and 5\% DMSO. To correct for contaminating ATPase activity, identical reactions were quenched with $100 \mu \mathrm{M}$ NVP-AUY922 and subtracted from unquenched reactions (DMSO control). In the titration experiments (Fig. 3), $1 \mu \mathrm{M}$ of Hsp82p was added to reactions containing either $1,2,4,8,12$, or $16 \mu \mathrm{M}$ of Ahalp, Ahalp ${ }^{\Delta 11}$, or $4 \mu \mathrm{M}$ of Hsp82p was added to reactions containing either $4,8,16$, or $32 \mu \mathrm{M}$ Hchlp or Hchlp ${ }^{\Delta 11}$. The ATPase assays were started by the addition of the regenerating system consisting of $\mathrm{MgCl}_{2}$, DTT, NADH, ATP, PEP, PK/LDH. Fit lines were calculated according to the following equation $\left(Y=\left(\left(B_{\max }{ }^{*} X\right) /\left(K_{\text {app }}+X\right)\right)+X_{0}\right)^{36}$. In the ATP titration experiment (Fig. 7 and S1), $2 \mu \mathrm{M}$ of Hsp82p was added to reactions containing 20 $\mu \mathrm{M}$ of either Ahalp or Ahalp $\mathrm{p}^{\Delta 11}$ or $4 \mu \mathrm{M}$ of Hsp82p was added to reactions containing $40 \mu \mathrm{M}$ Hchlp or Hchlp $\mathrm{p}^{\Delta 11}$. The regenerating system containing $\mathrm{MgCl}_{2}$, DTT, NADH, PEP, and PK/LDH was added to the reactions and the reaction was started by the addition of either $12.5,25,50,100,200,400,800$, or $1600 \mu \mathrm{M}$ of
ATP. ATPase rates were analyzed with the Michaelis-Menten non-linear regression function in GraphPad Prism. In the co-chaperone switching ATPase experiments (Fig. 3e), $4 \mu \mathrm{M}$ of indicated co-chaperones and buffers were added to the wells and allowed to mix for $10 \mathrm{~min} .2 \mu \mathrm{M}$ of Hsp82p was then added and allowed to mix for $10 \mathrm{~min}$ before starting the reaction through the addition of our regenerating system containing $\mathrm{MgCl}_{2}$, DTT, NADH, ATP, PEP, PK/LDH The ATPase rate shown in $\mu \mathrm{M}$ ATP hydrolyzed per minute per $\mu \mathrm{M}$ of Hsp82p $(1 / \mathrm{min})$.

PET fluorescence experiments. Double mutant S51C-A110W of yeast Hsp90 was synthesized using recombinant methods and modified with the fluorophore AttoOxa11 (AttoTec) as previously described ${ }^{34}$. Time-dependent fluorescence intensities were measured from Hsp90 samples in a quartz glass cuvette using a FP6500 spectrofluorimeter (Jasco). Fluorescence was excited at $620 \mathrm{~nm}$ and emission intensities were recorded at a wavelength of $678 \mathrm{~nm}$. Measurements were carried out at room temperature. Hsp90 samples were prepared in $50 \mathrm{mM}$ phosphate, $\mathrm{pH} 7.5$, with the ionic strength adjusted to $200 \mathrm{mM}$ using potassium chloride, containing $10 \mathrm{mM} \mathrm{MgCl}_{2}$ and $150 \mathrm{nM}$ of AttoOxal1-labeled Hsp90 construct. $5 \mu \mathrm{M}$ non-labeled wildtype Hsp90 protein was added and incubated at room temperature for $30 \mathrm{~min}$ to ensure that only one subunit in hetero-dimeric constructs carried the fluorophore. Aha-type co-chaperones were added at $20 \mu \mathrm{M}$ concentration. Reactions were started by addition of $2 \mathrm{mM}$ AMP-PNP. Fluorescence transients were analyzed by fitting exponential decay functions. Data of the Hsp90 sample without Aha-type co-chaperone required a bi-exponential function to fit them accurately, likely caused by molecular ground-state heterogeneity as discussed previously ${ }^{34}$. The reported time constant is the average time constant of two exponentials weighted by the respective, relative amplitudes. Data of Hsp90 samples pre-incubated with Aha-type co-chaperone fitted well to mono-exponential decay functions. Samples of Hsp90 in complex with Ahalp and Ahalp $\mathrm{p}^{11}$ showed a burst phase kinetics after the addition of AMP-PNP, which was faster than the time resolution of the experimental setup. The burst phase was caused by strong acceleration of lid closure caused by Ahalp and Ahalp ${ }^{\Delta 11}$. Values of $\tau$ are mean values of three measurements and errors are the standard deviation of these three measurements.

Reporting Summary. Further information on experimental design is available in the Nature Research Reporting Summary linked to this article.

\section{Data availability}

Data supporting the findings of this manuscript are available from the corresponding author upon reasonable request. A reporting summary for this Article is available as a Supplementary Information file. The source data underlying Figs. 3b-e, 4b, 4d, 5c, d, 6a, b, 7a, 9, and Supplementary Figs. 1 and 2 are provided as a Source Data file.

Received: 30 May 2018 Accepted: 1 March 2019

Published online: 20 March 2019

\section{References}

1. Eckl, J. M. \& Richter, K. Functions of the Hsp90 chaperone system: lifting client proteins to new heights. Int. J. Biochem. Mol. Biol. 4, 157-165 (2013).

2. Taipale, M. et al. Quantitative analysis of HSP90-client interactions reveals principles of substrate recognition. Cell 150, 987-1001 (2012).

3. Prodromou, C. et al. Regulation of Hsp90 ATPase activity by tetratricopeptide repeat (TPR)-domain co-chaperones. EMBO J. 18, 754-762 (1999).

4. Richter, K., Walter, S. \& Buchner, J. The Co-chaperone Sbal connects the ATPase reaction of Hsp90 to the progression of the chaperone cycle. J. Mol. Biol. 342, 1403-1413 (2004).

5. Siligardi, G. et al. Co-chaperone regulation of conformational switching in the Hsp90 ATPase cycle. J. Biol. Chem. 279, 51989-51998 (2004).

6. Li, J., Soroka, J. \& Buchner, J. The Hsp90 chaperone machinery: conformational dynamics and regulation by co-chaperones. Biochim. Biophys. Acta. 1823, 624-635 (2012).

7. Armstrong, H., Wolmarans, A., Mercier, R., Mai, B. \& LaPointe, P. The co-chaperone Hch1 regulates Hsp90 function differently than its homologue Ahal and confers sensitivity to yeast to the Hsp90 inhibitor NVP-AUY922. PLoSONE 7, e49322 (2012).

8. Lee, C. T., Graf, C., Mayer, F. J., Richter, S. M. \& Mayer, M. P. Dynamics of the regulation of Hsp90 by the co-chaperone Stil. EMBO J. 31, 1518-1528 (2012).

9. Li, J., Richter, K., Reinstein, J. \& Buchner, J. Integration of the accelerator Ahal in the Hsp90 co-chaperone cycle. Nat. Struct. Mol. Biol. 20, 326-331 (2013).

10. Chang, H. C., Nathan, D. F. \& Lindquist, S. In vivo analysis of the Hsp90 cochaperone Sti1 (p60). Mol. Cell. Biol. 17, 318-325 (1997). 
11. Fang, Y., Fliss, A. E., Rao, J. \& Caplan, A. J. SBA1 encodes a yeast hsp90 cochaperone that is homologous to vertebrate p23 proteins. Mol. Cell. Biol. 18 , 3727-3734 (1998).

12. Knoblauch, R. \& Garabedian, M. J. Role for Hsp90-associated cochaperone p23 in estrogen receptor signal transduction. Mol. Cell. Biol. 19, 3748-3759 (1999).

13. Panaretou, B. et al. Activation of the ATPase activity of hsp 90 by the stressregulated cochaperone aha1. Mol. Cell 10, 1307-1318 (2002).

14. Richter, K. \& Buchner, J. hsp90: twist and fold. Cell 127, 251-253 (2006)

15. Prodromou, C. The 'active life' of Hsp90 complexes. Biochim. Biophys. Acta 1823, 614-623 (2012).

16. Obermann, W. M., Sondermann, H., Russo, A. A., Pavletich, N. P. \& Hartl, F. $\mathrm{U}$. In vivo function of Hsp90 is dependent on ATP binding and ATP hydrolysis. J. Cell. Biol. 143, 901-910 (1998).

17. Panaretou, B. et al. ATP binding and hydrolysis are essential to the function of the Hsp90 molecular chaperone in vivo. EMBO J. 17, 4829-4836 (1998).

18. Lotz, G. P., Lin, H., Harst, A. \& Obermann, W. M. Aha1 binds to the middle domain of Hsp90, contributes to client protein activation, and stimulates the ATPase activity of the molecular chaperone. J. Biol. Chem. 278, 17228-17235 (2003).

19. Meyer, P. et al. Structural basis for recruitment of the ATPase activator Aha1 to the Hsp90 chaperone machinery. EMBO J. 23, 1402-1410 (2004).

20. Wang, X. et al. Hsp90 cochaperone Ahal downregulation rescues misfolding of CFTR in cystic fibrosis. Cell 127, 803-815 (2006).

21. Harst, A., Lin, H. \& Obermann, W. M. Aha1 competes with Hop, p50 and p23 for binding to the molecular chaperone Hsp90 and contributes to kinase and hormone receptor activation. Biochem. J. 387, 789-796 (2005).

22. Hawle, P. et al. The middle domain of Hsp90 acts as a discriminator between different types of client proteins. Mol. Cell. Biol. 26, 8385-8395 (2006).

23. Holmes, J. L., Sharp, S. Y., Hobbs, S. \& Workman, P. Silencing of HSP90 cochaperone AHA1 expression decreases client protein activation and increases cellular sensitivity to the HSP90 inhibitor 17-allylamino-17demethoxygeldanamycin. Cancer Res. 68, 1188-1197 (2008).

24. Shelton, L. B. et al. Hsp90 activator Ahal drives production of pathological tau aggregates. Proc. Natl Acad. Sci. USA 114, 9707-9712 (2017).

25. Ghaemmaghami, S. et al. Global analysis of protein expression in yeast. Nature 425, 737-741 (2003).

26. Sahasrabudhe, P., Rohrberg, J., Biebl, M. M., Rutz, D. A. \& Buchner, J. The plasticity of the Hsp90 Co-chaperone system. Mol. Cell 67, 947-961 e5 (2017).

27. Bohen, S. P. Genetic and biochemical analysis of $\mathrm{p} 23$ and ansamycin antibiotics in the function of Hsp90-dependent signaling proteins. Mol. Cell. Biol. 18, 3330-3339 (1998).

28. Carrigan, P. E. et al. Multiple domains of the co-chaperone Hop are important for Hsp70 binding. J. Biol. Chem. 279, 16185-16193 (2004).

29. Nelson, G. M. et al. The heat shock protein 70 cochaperone hip enhances functional maturation of glucocorticoid receptor. Mol. Endocrinol. 18, 1620-1630 (2004).

30. Horvat, N. K. et al. A mutation in the catalytic loop of Hsp90 specifically impairs ATPase stimulation by Ahalp, but not Hch1p. J. Mol. Biol. 426, 2379-2392 (2014).

31. Pearl, L. H. Review: The HSP90 molecular chaperone-an enigmatic ATPase Biopolymers 105, 594-607 (2016).

32. Mayer, M. P. \& Le Breton, L. Hsp90: breaking the symmetry. Mol. Cell 58, 8-20 (2015).

33. Ali, M. M. et al. Crystal structure of an Hsp90-nucleotide-p23/Sbal closed chaperone complex. Nature 440, 1013-1017 (2006).

34. Schulze, A. et al. Cooperation of local motions in the Hsp90 molecular chaperone ATPase mechanism. Nat. Chem. Biol. 12, 628-635 (2016).

35. Koulov, A. V. et al. Biological and structural basis for Ahal regulation of Hsp90 ATPase activity in maintaining proteostasis in the human disease cystic fibrosis. Mol. Biol. Cell 21, 871-884 (2010).

36. Retzlaff, M. et al. Asymmetric activation of the hsp90 dimer by its cochaperone aha1. Mol. Cell 37, 344-354 (2010).

37. Wolmarans, A., Lee, B., Spyracopoulos, L. \& LaPointe, P. The mechanism of Hsp90 ATPase stimulation by Aha1. Sci. Rep. 6, 33179 (2016).

38. Zuehlke, A. D. et al. An Hsp90 co-chaperone protein in yeast is functionally replaced by site-specific posttranslational modification in humans. Nat. Commun. 8, 15328 (2017).

39. Zierer, B. K. et al. Importance of cycle timing for the function of the molecular chaperone Hsp90. Nat. Struct. Mol. Biol. 23, 1020-1028 (2016).

40. Ali, J. A., Jackson, A. P., Howells, A. J. \& Maxwell, A. The 43-kilodalton Nterminal fragment of the DNA gyrase B protein hydrolyzes ATP and binds coumarin drugs. Biochemistry 32, 2717-2724 (1993).

41. Wuest, F., Bouvet, V., Mai, B. \& LaPointe, P. Fluorine- and rheniumcontaining geldanamycin derivatives as leads for the development of molecular probes for imaging Hsp90. Org. Biomol. Chem. 10, 6724-6731 (2012).
42. Ericsson, U. B., Hallberg, B. M., Detitta, G. T., Dekker, N. \& Nordlund, P. Thermofluor-based high-throughput stability optimization of proteins for structural studies. Anal. Biochem. 357, 289-298 (2006).

43. Lavinder, J. J., Hari, S. B., Sullivan, B. J. \& Magliery, T. J. High-throughput thermal scanning: a general, rapid dye-binding thermal shift screen for protein engineering. J. Am. Chem. Soc. 131, 3794-3795 (2009).

44. Nathan, D. F., Vos, M. H. \& Lindquist, S. Identification of SSF1, CNS1, and $\mathrm{HCH} 1$ as multicopy suppressors of a Saccharomyces cerevisiae Hsp90 loss-of-function mutation. Proc. Natl Acad. Sci. USA 96, 1409-1414 (1999).

45. Graf, C., Stankiewicz, M., Kramer, G. \& Mayer, M. P. Spatially and kinetically resolved changes in the conformational dynamics of the Hsp90 chaperone machine. EMBO J. 28, 602-613 (2009).

46. Hessling, M., Richter, K. \& Buchner, J. Dissection of the ATP-induced conformational cycle of the molecular chaperone Hsp90. Nat. Struct. Mol. Biol. 16, 287-293 (2009).

47. Atkins, G. L. \& Nimmo, I. A. Current trends in the estimation of Michaelis-Menten parameters. Anal. Biochem. 104, 1-9 (1980).

48. Schnell, S. Validity of the Michaelis-Menten equation-steady-state or reactant stationary assumption: that is the question. FEBS J. 281, 464-472 (2014).

49. Graf, C., Lee, C. T., Eva Meier-Andrejszki, L., Nguyen, M. T. \& Mayer, M. P. Differences in conformational dynamics within the Hsp90 chaperone family reveal mechanistic insights. Front. Mol. Biosci. 1, 4 (2014).

50. Richter, K. et al. Intrinsic inhibition of the Hsp90 ATPase activity. J. Biol. Chem. 281, 11301-11311 (2006).

51. Weikl, T. et al. C-terminal regions of Hsp90 are important for trapping the nucleotide during the ATPase cycle. J. Mol. Biol. 303, 583-592 (2000).

52. Richter, K., Muschler, P., Hainzl, O. \& Buchner, J. Coordinated ATP hydrolysis by the Hsp90 dimer. J. Biol. Chem. 276, 33689-33696 (2001).

53. Richter, K. et al. Conserved conformational changes in the ATPase cycle of human Hsp90. J. Biol. Chem. 283, 17757-17765 (2008).

54. Krukenberg, K. A., Street, T. O., Lavery, L. A. \& Agard, D. A. Conformational dynamics of the molecular chaperone Hsp90. Q. Rev. Biophys. 44, 229-255 (2011)

55. Soroka, J. et al. Conformational switching of the molecular chaperone Hsp90 via regulated phosphorylation. Mol. Cell 45, 517-528 (2012).

56. Hainzl, O., Lapina, M. C., Buchner, J. \& Richter, K. The charged linker region is an important regulator of Hsp90 function. J. Biol. Chem. 284, 22559-22567 (2009).

57. Halpin, J. C. \& Street, T. O. Hsp90 sensitivity to ADP reveals hidden regulation mechanisms. J. Mol. Biol. 429, 2918-2930 (2017).

58. LaPointe, P., Wei, X. \& Gariepy, J. A role for the protease-sensitive loop region of Shiga-like toxin 1 in the retrotranslocation of its A1 domain from the endoplasmic reticulum lumen. J. Biol. Chem. 280, 23310-23318 (2005).

59. Mumberg, D., Muller, R. \& Funk, M. Yeast vectors for the controlled expression of heterologous proteins in different genetic backgrounds. Gene 156, 119-122 (1995).

60. Taxis, C. \& Knop, M. System of centromeric, episomal, and integrative vectors based on drug resistance markers for Saccharomyces cerevisiae. Biotechniques 40, 73-78 (2006).

61. Nathan, D. F. \& Lindquist, S. Mutational analysis of Hsp90 function: interactions with a steroid receptor and a protein kinase. Mol. Cell. Biol. 15, 3917-3925 (1995).

62. Kravats, A. N. et al. Functional and physical interaction between yeast Hsp90 and Hsp70. Proc. Natl Acad. Sci. USA 115, E2210-E2219 (2018).

63. Murphy, S. M., Bergman, M. \& Morgan, D. O. Suppression of c-Src activity by C-terminal Src kinase involves the c-Src $\mathrm{SH} 2$ and $\mathrm{SH} 3$ domains: analysis with Saccharomyces cerevisiae. Mol. Cell. Biol. 13, 5290-5300 (1993)

64. Flom, G. A., Langner, E. \& Johnson, J. L. Identification of an Hsp90 mutation that selectively disrupts cAMP/PKA signaling in Saccharomyces cerevisiae. Curr. Genet. 58, 149-163 (2012).

65. Evan, G. I., Lewis, G. K., Ramsay, G. \& Bishop, J. M. Isolation of monoclonal antibodies specific for human c-myc proto-oncogene product. Mol. Cell. Biol. 5, 3610-3616 (1985).

\section{Acknowledgements}

We would like to thank Gary Eitzen (University of Alberta) for the anti-actin antibody. Work in the laboratory of P.L. was supported by funding from the Natural Sciences and Engineering Research Council of Canada (386803), the Canadian Institutes of Health Research (97870), and Alberta Innovates Health Solutions (200900500). R.M. is supported by fellowships from the Alberta Cancer Foundation and Alberta Innovates Health Solutions. Work in the laboratory of J.L.J. was supported by funding from the National Institutes of Health (P30 GM103324). 


\section{Author contributions}

R.M. designed and carried out experiments, and edited the manuscript. A.W. and J.S carried out experiments. H.N. designed experiments and edited the manuscript. J.L.J. identified the S25P yeast strain and edited the manuscript. P.L. conceived the study, designed experiments, and wrote the manuscript.

\section{Additional information}

Supplementary Information accompanies this paper at https://doi.org/10.1038/s41467019-09299-3.

Competing interests: The authors declare no competing interests.

Reprints and permission information is available online at http://npg.nature.com/ reprintsandpermissions/

Journal peer review information: Nature Communications thanks Cara Vaughan and the other anonymous reviewer(s) for their contribution to the peer review of this work. Peer reviewer reports are available.
Publisher's note: Springer Nature remains neutral with regard to jurisdictional claims in published maps and institutional affiliations.

(c) (i) Open Access This article is licensed under a Creative Commons Attribution 4.0 International License, which permits use, sharing adaptation, distribution and reproduction in any medium or format, as long as you give appropriate credit to the original author(s) and the source, provide a link to the Creative Commons license, and indicate if changes were made. The images or other third party material in this article are included in the article's Creative Commons license, unless indicated otherwise in a credit line to the material. If material is not included in the article's Creative Commons license and your intended use is not permitted by statutory regulation or exceeds the permitted use, you will need to obtain permission directly from the copyright holder. To view a copy of this license, visit http://creativecommons.org/ licenses/by/4.0/.

(C) The Author(s) 2019 